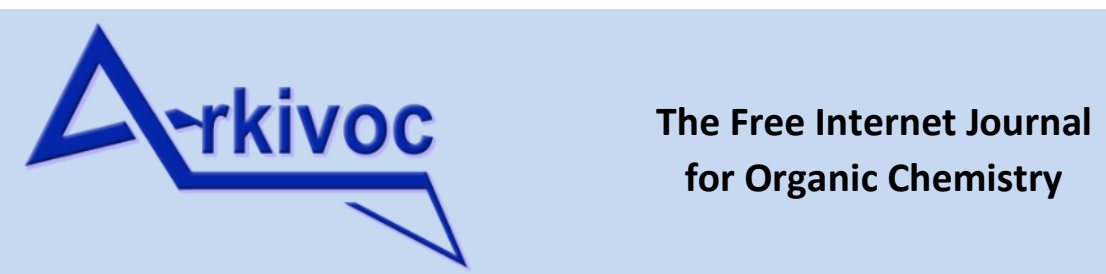

Archive for

Organic Chemistry

\title{
Review
}

Arkivoc 2018, part i, 348-382

\section{Synthesis of heterocycles via aerobic oxidation}

\begin{abstract}
Carlo Sambiagio
Department of Chemical Engineering and Chemistry, Micro Flow Chemistry \& Synthetic Methodology, Eindhoven University of Technology, Den Dolech 2, 5612 AZ Eindhoven, The Netherlands Email: C.Sambiagio@tue.nI
\end{abstract}

Received 12-11-2018

Accepted 01-26-2019

Published on line 03-03-2019

\section{Abstract}

Research in the field of aerobic oxidation has recently gained much attention from both academia and industry. The main reason for this has to be found in the inherent greenness of such methods. Although much investigation has been undertaken to develop new methods and reactions and to elucidate the mechanistic aspects of this chemistry, its applications in the synthesis of heterocycles have been relatively limited. In this review, the recent developments in this field are summarized.

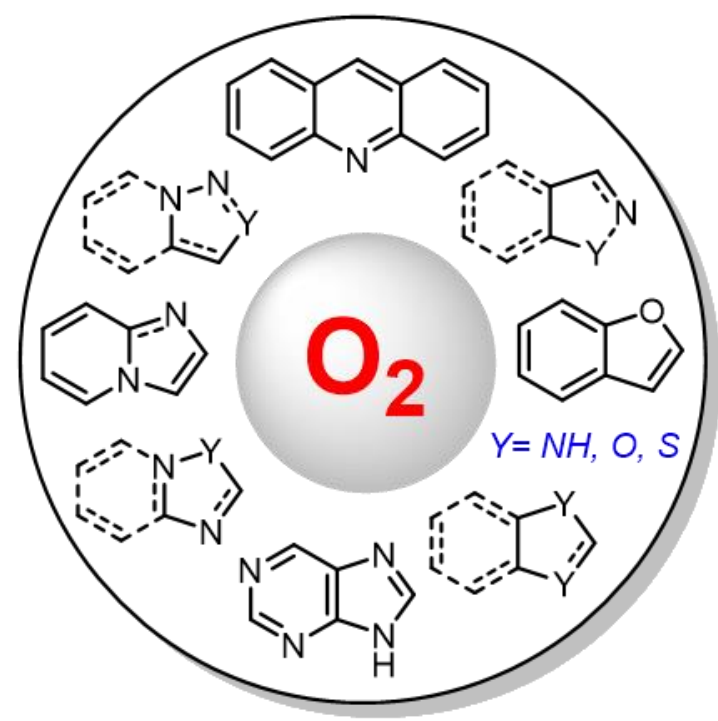

Keywords: Aerobic oxidation, heterocyclic synthesis, reaction mechanism, oxidative couplings, oxygen 


\section{Table of Contents}

1. Introduction

2. $\mathrm{Csp}^{3}-\mathrm{H}$ bond Oxygenation

2.1 Oxygenation / condensation with amines

2.2 Oxygenation / C-O bond formation

3. Oxidative Isocyanide Insertion

3.1 Formation of C-C + C-heteroatom bonds

3.2 Formation of two C-heteroatom bonds

3.3 Isocyanide insertion / acyl migration

4. Oxidative $\mathrm{CO}$ Insertion

5. Oxidative Friedlander annulation - Acridine Synthesis

6. Oxidative $\mathrm{N}$-Heteroatom Bond Formation

6.1 Oxidative $\mathrm{N}-\mathrm{N}$ bond formation

6.1.1 Synthesis of pyrazoles and indazoles

6.1.2 Synthesis of triazoles

6.2 Oxidative $\mathrm{N}-\mathrm{O}, \mathrm{N}-\mathrm{S}$, and $\mathrm{N}-\mathrm{Se}$ bond formation

7. Oxidative C-Heteroatom Bond Formation

7.1 Oxidative $\mathrm{C}-\mathrm{N}$ bond formation

7.2 Oxidative $\mathrm{C}-\mathrm{O}$ and $\mathrm{C}-\mathrm{S}$ bond formation

8. Intermolecular Oxidative Cyclization with Unsaturated Moieties

8.1 Synthesis of imidazole derivatives via cyclization of amidines with electron-poor alkenes

8.2 Synthesis of benzofurans via cyclization of phenols with alkynes

8.3 Miscellaneous cyclizations with alkynes

9. Conclusions

\section{Introduction}

Aerobic oxidation chemistry (i.e. the use of $\mathrm{O}_{2}$ as terminal oxidant in a chemical reaction) is a research field for which a steadily growing interest has been observed during the last 2 decades (Figure 1 , left). ${ }^{1-2}$ The increasing interest in green and sustainable chemistry from both academia and industry surely has an important role in this. As oxidations (in a general sense) are widespread reactions, the possibility to use an abundant, cheap, benign, and atom economic reagent for these transformations is a considerable motivation for all researchers in this field.

Due to its clear advantages, $\mathrm{O}_{2}$ has long been employed in several industrial processes, mostly for the production of bulk chemicals, such as cumene hydroperoxide (for the production of phenol and acetone) and cyclohexanone (production of polyamides). ${ }^{3}$ For these compounds, produced in very large scale and with a low market price, the use of oxygen is the only economically viable option. However, the employment of $\mathrm{O}_{2}$ in chemical processes also has several drawbacks, which somewhat limit its applications. The inefficient masstransfer of the gas into solution calls for high pressures to be used to speed up the reaction. On the other hand, a high $\mathrm{O}_{2}$ pressure in the presence of a flammable solvent at high temperatures represents an important safety hazard. Many aerobic industrial processes for the synthesis of bulk chemicals, therefore, utilize synthetic air (5$10 \% \mathrm{O}_{2}$ in $\mathrm{N}_{2}$ ) instead of pure $\mathrm{O}_{2}$. The low reaction rate achieved in this way (due to the low concentration of 
oxygen) is generally compensated by the implementation of recycling streams at the end of the reaction, overall achieving a good compromise between reaction rate/conversion, safety, and cost. While this is generally a good solution for the production of bulk chemicals, which can count on dedicated plant reactors, ${ }^{2-4}$ this is not the case for the fine chemical and pharmaceutical industry, where the smaller scale does not justify a dedicated plant, and the higher price of the final products also allows the use of more expensive, but easier to handle and process-safer oxidants. ${ }^{4}$ Nonetheless, as most other oxidant are also characterized by a low atom economy, high content in metals, and a certain toxicity, now research is being carried out on the development of alternative methods to utilize $\mathrm{O}_{2}$ in a more controlled and safer manner, so to allow its use even in the fine chemicals and pharma industry. For example, continuous-flow chemistry in microreactors is now being often employed to improve mass transfer between gas and liquid, and reduce ignition hazard, due to the low concentration of oxygen in every segment of the flow reactor, and the absence of a headspace filled with pressurized gas. ${ }^{4-6}$ These improvements will on the long run make aerobic oxidation methods more attractive and ultimately broaden their applications.

Aerobic oxidations can be broadly divided into oxygenase- and oxidase-type reactions, which take their name from the corresponding enzymes that catalyze these processes in biological systems. Simply put, in oxygenase chemistry the $\mathrm{O}_{2}$ acts as a reactant, and oxygen atoms are incorporated into the molecule (either an intermediate or the final product). For example, on $\mathrm{C}-\mathrm{H}$ bonds, oxygenase results in the formation of alcohols, ketones/aldehydes, or carboxylic acids. In oxidase chemistry, the oxygen only acts as an electron acceptor, and no oxygen atom is incorporated into the product. For example, oxidase processes occur when a metal catalyst, at the end of a catalytic cycle in an inactive state (low oxidation state), is re-oxidized to its active form (higher oxidation state) by donating electrons to $\mathrm{O}_{2}$. This is typically the case in, for example, cross-dehydrogenative couplings (CDCs) between two R-H moieties to form R-R bonds. Between these two reactivity modes, aerobic oxidations can in theory be applied to the formation of virtually any type of C-C, C-heteroatom, or heteroatomheteroatom bonds. This versatility is a great advantage in synthesis, and aerobic oxidation methods have been applied to a large number of reactions in the last decades, in particular alcohol dehydrogenation, oxygenation of methyl/methylene groups to carbonyl derivatives, and inter- and intramolecular CDCs.

Among its applications, the synthesis of heterocycles is particularly attractive, because of the variety of bonds that can be formed via aerobic oxidation strategies within the same heterocyclic core. Due to the importance of heterocycles in organic, inorganic, material, and medicinal chemistry, the development of novel retrosynthetic pathways for their synthesis, or the improvement of existing pathways into greener alternatives, is of high interest. Although this specific sub-area of aerobic oxidation has not been extensively explored to date, an increasing number of publications in the field has been observed from the first decade of the 2000's, with peaks in the period 2012-2014 (Figure 1, right). This trend reflects an increasing interest from the chemistry community, and further exploration of the field is expected in the near future. This review summarizes the recent advancements (since approximately 2010) on the use of $\mathrm{O}_{2}$ as oxidant in heterocyclic synthesis. In this review the literature will not be divided according to the heterocyclic cores formed, as many strategies can be applied to the synthesis of a variety of heterocycles. Instead, the main different strategies will be discussed separately, showing all the possibilities offered in synthesis by each specific approach. 

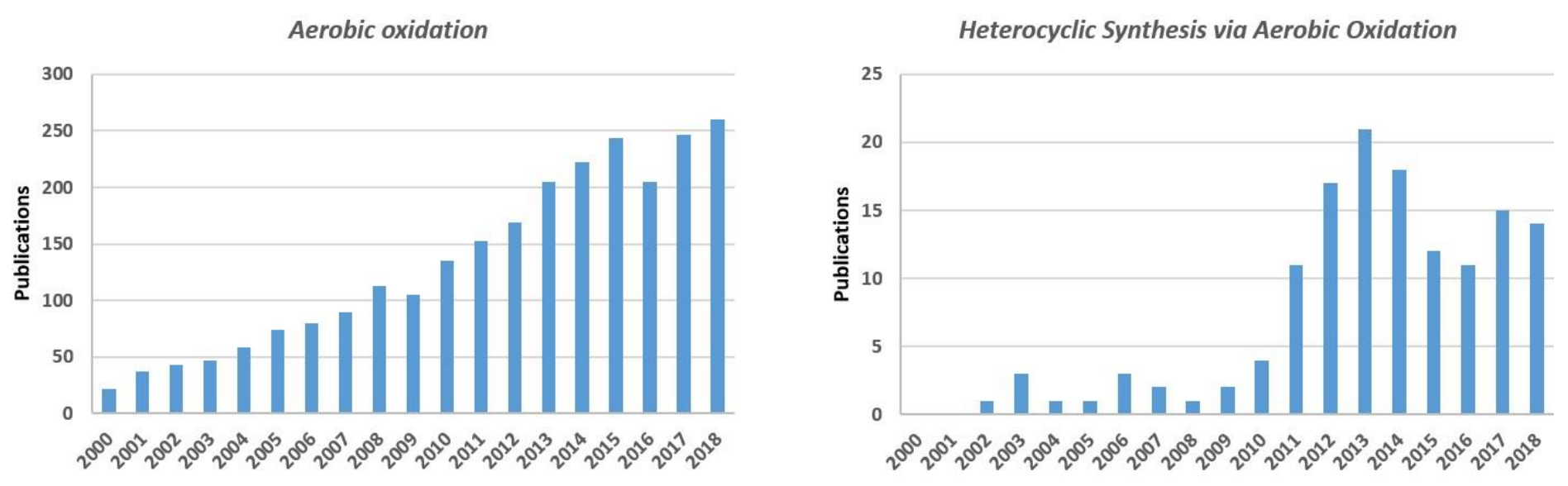

Figure 1. Left: publications containing the words "aerobic oxidation" in the period 2000-2018, SciFinder search $7^{\text {th }}$ Jan. 2019. Right: publications containing the concepts "heterocycle synthesis" and "aerobic oxidation" associated in the period 2000-2018, SciFinder search $7^{\text {th }}$ Jan. 2019.

\section{Csp ${ }^{3}-\mathrm{H}$ bond Oxygenation}

While many of the aerobic processes for the synthesis of heterocycles involve oxidase-type mechanisms, one common exception is the oxygenation of $\mathrm{Csp}^{3}-\mathrm{H}$ bonds as a crucial step in the formation of the heterocycle. Two strategies can be in particular distinguished: the oxidation of $\mathrm{C}-\mathrm{H}$ bonds to carbonyls, followed by in-situ condensation with an amine (new $\mathrm{C}-\mathrm{N}$ bond in the heterocycle); and the oxygenation of $\mathrm{C}-\mathrm{H}$ bonds, followed by radical coupling of the newly installed oxygen with activated carbon moieties, leading to new $\mathrm{C}-\mathrm{O}$ bonds.

\subsection{Oxygenation / condensation with amines}

The condensation of a carbonyl and an amine to give an imine is a common reaction encountered in the synthesis of aza-heterocycles. An interesting approach is the in-situ formation of the carbonyl reactant from the corresponding precursors. The formation of ketones or aldehydes, or analogous intermediates, can be directly achieved via Cu-/Fe-catalyzed aerobic oxidation of alcohols (oxidase) or direct oxygenation of activated (e.g. benzylic) methylenes (oxygenase), according to Scheme 1.

Another possible method is the iodine-catalyzed Kornblum oxidation, which provides access to carbonyls from the corresponding methylenes in an indirect way. In this case the oxygen atom in the carbonyl comes from DMSO. Being an indirect oxidation, heterocyclic synthesis involving this process will not be treated here. ${ }^{7-8}$

The carbonyl obtained under aerobic conditions can then be transformed in-situ into an imine. In the synthesis of heterocycles, this step generally does not constitute the ring-closing step, so that this process is intermolecular, and is typically followed by an intramolecular nucleophilic attack to the imine carbon or a cyclization at the methylene alpha to the nitrogen, furnishing the desired aza-heterocycle (Scheme 1). 


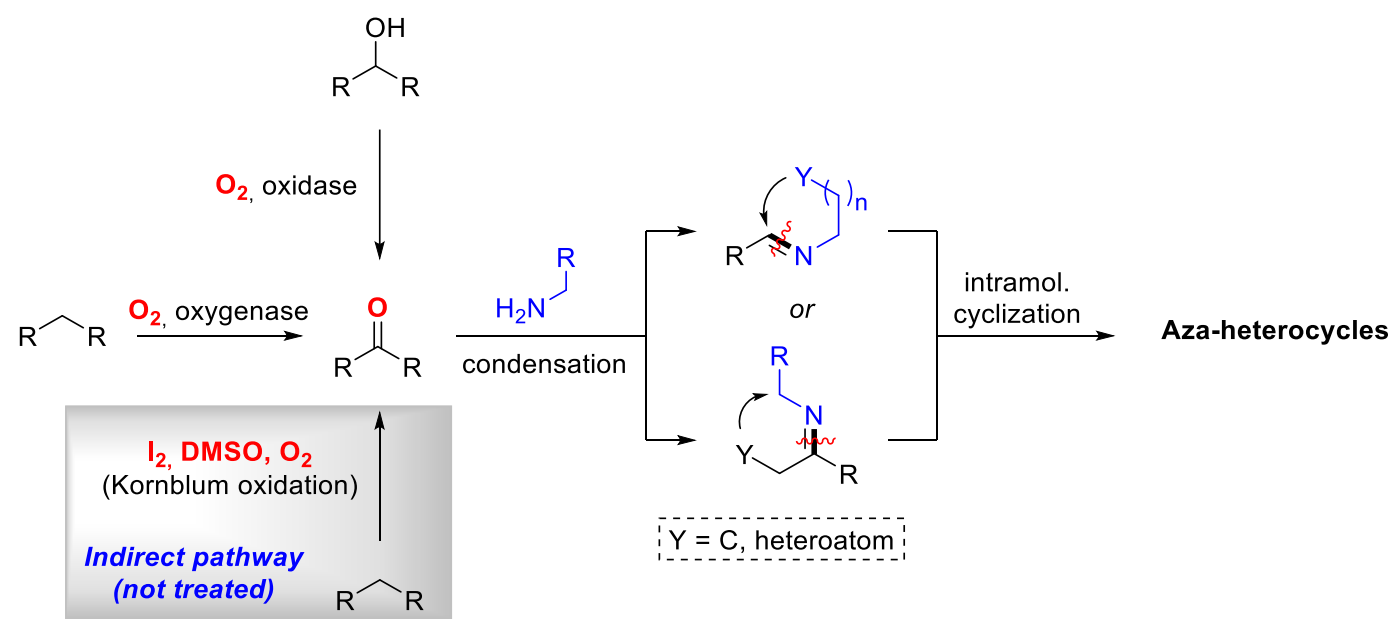

Scheme 1. Possible pathways for the synthesis of aza-heterocyclic cores via $\mathrm{Csp}^{3}-\mathrm{H}$ oxygenation followed by condensation with amines.

The synthesis of quinazolinones and other diheterocycles (2-5) has been reported by Zhou/Yin making use of 2methylaza-heterocycles ${ }^{9}$ or phenylacetic acids ${ }^{10}$ as aldehyde precursors, according to

Scheme 2A-B. While the first method is more atom economic, it has the limitation of only working on 2methylaza-arenes as aldehyde precursors, due to its mechanism (see

Scheme 2, bottom). ${ }^{9,11}$ The second method required prefunctionalized, though easily accessible, precursors, but a larger variety of aryl and heteroaryl substituents could be employed. ${ }^{10} \mathrm{~A}$ variety of other oxidants tested with this methods resulted in no reaction.

The use of 2-methylaza-arenes was also reported by Yan for the synthesis of functionalized quinolines $\mathbf{8}$ and pyrroloquinoxalines 9 (

Scheme 2C). ${ }^{12}$ Very notably, it was shown that apart from 2-methylpyridines and 2-methylquinolines, also 4-methylpyridine was effective in the reaction, although 4-methylquinoline was not. In this protocol, after the formation of the imine moiety, an electrocyclization reaction takes place, leading to ring closure. Aromaticity is then re-established by oxidation.

While this method is limited to activated picolines as reactants, the introduction of an alkyl chain in the pyrroloquinoxaline scaffold is possible using aliphatic alcohols as reactants. These are oxidized to the corresponding aliphatic aldehyde, which undergo cyclisation giving the desired scaffold 10. The aerobic oxidation of $\mathrm{N}$-aryl pyrroles 7 with alcohols has been reported using superstoichiometric Fe, ${ }^{13}$ but noteworthy is the metal-free version reported by Jiang in 2017 (

Scheme 2D). ${ }^{14}$

The mechanism of the Cu-catalyzed oxidation of 2-methylpyridines has been studied in details by Maes and co-workers, ${ }^{11}$ and is at the base of the lack of reactivity of 3-methylpyridines observed by Zhou/Yin. ${ }^{9}$ The reaction is initiated by an imine-enamine tautomerism, which cannot occur in the 3-methyl isomer. Reaction of the enamine tautomer with $\mathrm{Cu} / \mathrm{O}_{2}$ results in the formation of the aldehyde intermediate, or a peroxide intermediate which directly reacts with the amine. ${ }^{9}$ The mechanism of the decarboxylative protocol is suggested to occur via aerobic oxidation of the methylene alpha to the carboxyl moiety, followed by decarboxylation, leading to the corresponding benzaldehydes (

Scheme 2, bottom). ${ }^{10}$ 
A<smiles>[R]NC(=O)c1ccccc1N</smiles>

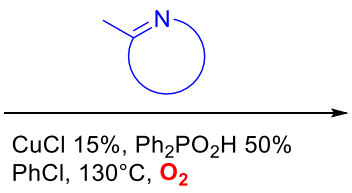

$\mathrm{PhCl}, 130^{\circ} \mathrm{C}, \mathrm{O}_{2}$

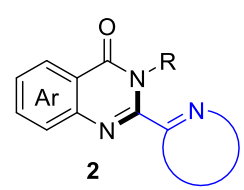

18 examples, up to $92 \%$ yield
$\mathrm{O}_{2}$ vs other oxidants

$\mathrm{O}_{2} \quad 80 \%$

$\mathrm{R}=\mathrm{H}, \mathrm{Me}$

azaheterocycle $=$ pyridines, pyrazine, quinolines, $($ benzo)thiazole<smiles>[H][Y]c1ccccc1NN</smiles><smiles>[X]c1ccccc1N</smiles>

$\mathrm{R}=\mathrm{H}, \mathrm{Me}$

$\mathrm{Ar}^{1}=$ substituted $\mathrm{Ph}$, heterocycles

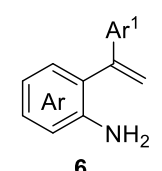

C

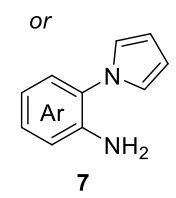

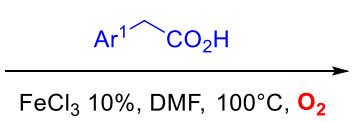<smiles>[R]n1c([AlH2])nc2ccccc2c1=O</smiles>

3

18 examples, up to $92 \%$ yield

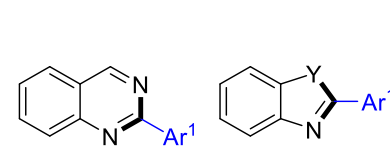

\section{4}

9 examples, up to $90 \%$ yield (air as oxidant) up to $91 \%$ yield
$(\mathrm{Y}=\mathrm{NH}, \mathrm{S})$

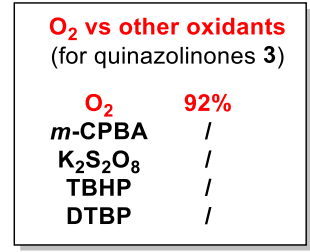

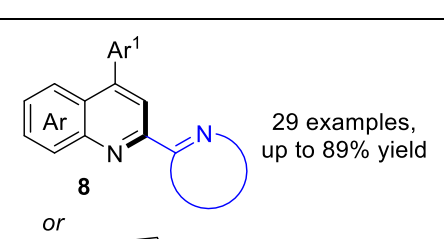

$\overrightarrow{\mathrm{CuCl}_{2} \cdot 2 \mathrm{H}_{2} \mathrm{O} 10 \%, \mathrm{DMF}, 130^{\circ} \mathrm{C}, \mathrm{O}_{2}}$

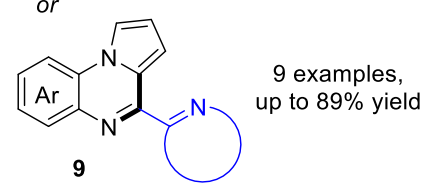
$\mathrm{O}_{2}$ vs other oxidants (for quinolines 8)
$\mathrm{O}_{2} \quad 71 \%$ air $59 \%$

azaheterocycle $=$ quinolines, pyridines (also 4-methyl pyridine)

D<smiles>Nc1ccccc1-n1ccc2ccccc21</smiles>

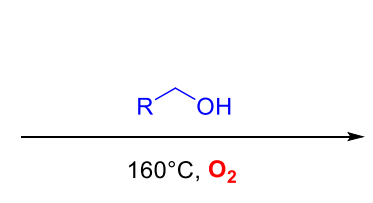

$160^{\circ} \mathrm{C}, \mathrm{O}_{2}$

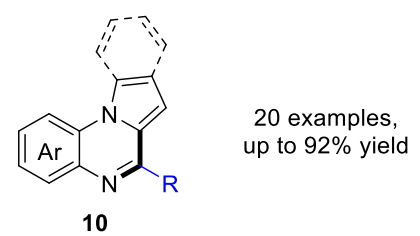

$\mathrm{R}=\mathrm{H}$, alkyl

Simplified mechanism of the oxidation of 2-methylaza-arenes:

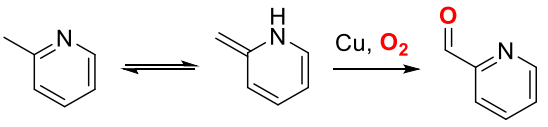

- Simplified mechanism of the oxidation of phenylacetic acids:<smiles>CC(C)Cc1cccc(C(=O)C(=O)OCc2ccc(CC(=O)O)cc2)c1</smiles>

Scheme 2. Synthesis of aza-heterocycles via $\mathrm{Csp}^{3}$-H oxygenation / condensation starting from picolines or phenylacetic acids.

When benzylic amines are used, a different mechanism becomes available. In such cases, the benzylic carbon of the amine can be involved in an intramolecular oxidative cyclization, furnishing the heterocycle. The synthesis of imidazole-based compounds 11-13 was reported via this mechanism by Zhang and Ji/Wang ( Scheme 3). ${ }^{15-16}$ 


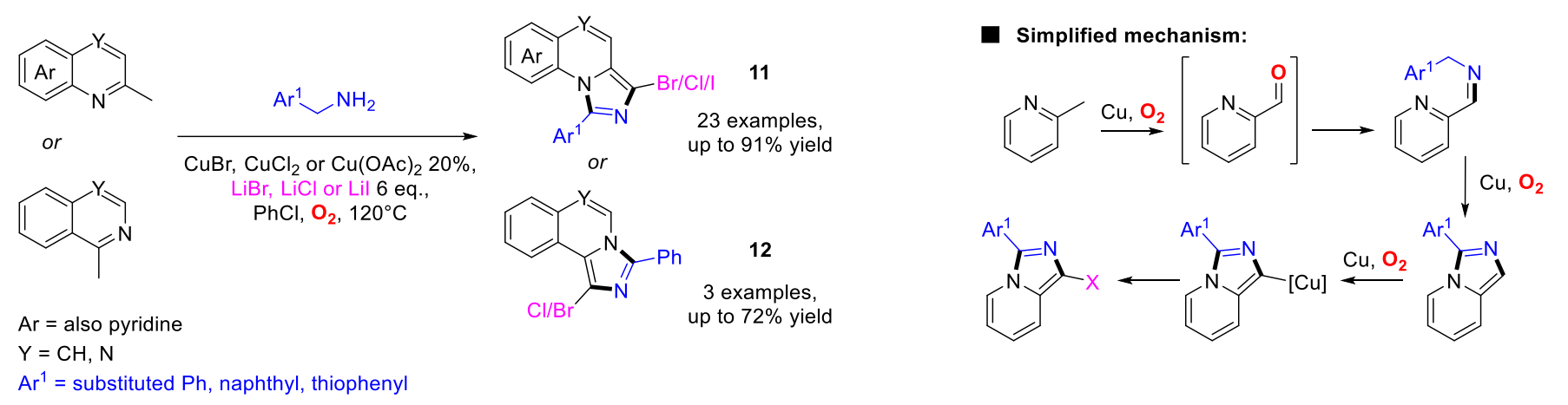

$\mathrm{r}^{\mathrm{1}}=$ substituted $\mathrm{Ph}$, naphthyl, thiopheny

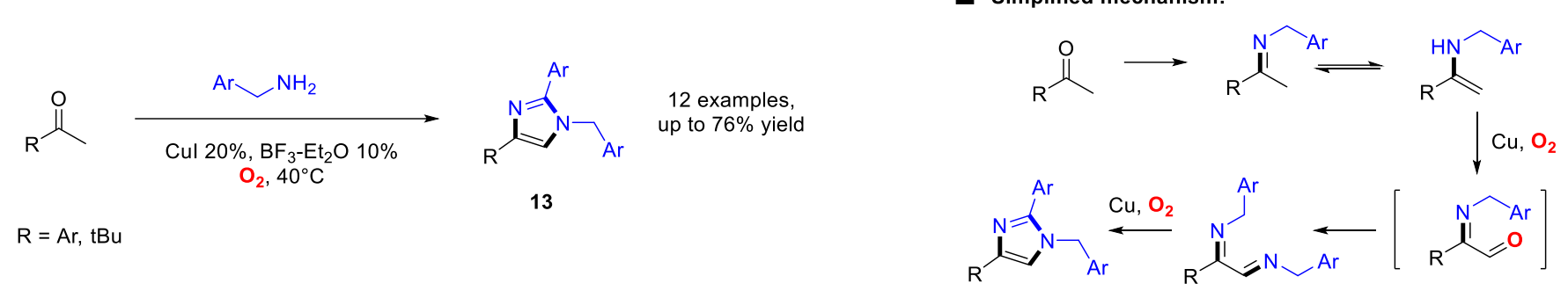

Scheme 3. Synthesis of aza-heterocycles via $\mathrm{Csp}^{3}-\mathrm{H}$ oxygenation / condensation involving benzylamines as reactants.

\subsection{Oxygenation / C-O bond formation}

Although the formation of imines in-situ from carbonyls is a particularly recurrent strategy for the synthesis of heterocycles under aerobic conditions, oxygenase methods can also be applied to the synthesis of O-containing heterocycles, where the aerobic oxygen remains in the final product. Chiba and co-workers were able to synthesize a range of dihydrooxazoles 16 from $\mathrm{N}$-alkylamidines 14 via a Cu-catalyzed process (

Scheme 4A). ${ }^{17}$ The reaction occurred via oxidation of the amidine moiety, generating a nitrogen radical, followed by a 1,5-hydrogen radical shift, leading to a tertiary carbon radical. Cu-catalyzed oxygenation in this position and subsequent cyclization resulted in the dihydrooxazoles 16. 1,3-Benzooxazines 17 could be prepared using the same strategy from $\mathrm{N}$-(2-isopropylphenyl)amidines 15 (

Scheme $4 A) .{ }^{17}$ In this case a 1,6-hydrogen radical shift was postulated for the formation of the intermediate carbon radical.

Related strategies were developed by Jiao et al. for the synthesis of di- or trisubstituted oxazoles 18-19. ${ }^{18-19}$ Both protocols involve the formation of imine intermediates, followed by oxygenation at the alpha position and final cyclization-dehydrogenation to furnish the oxazole core (

Scheme 4B-C). 

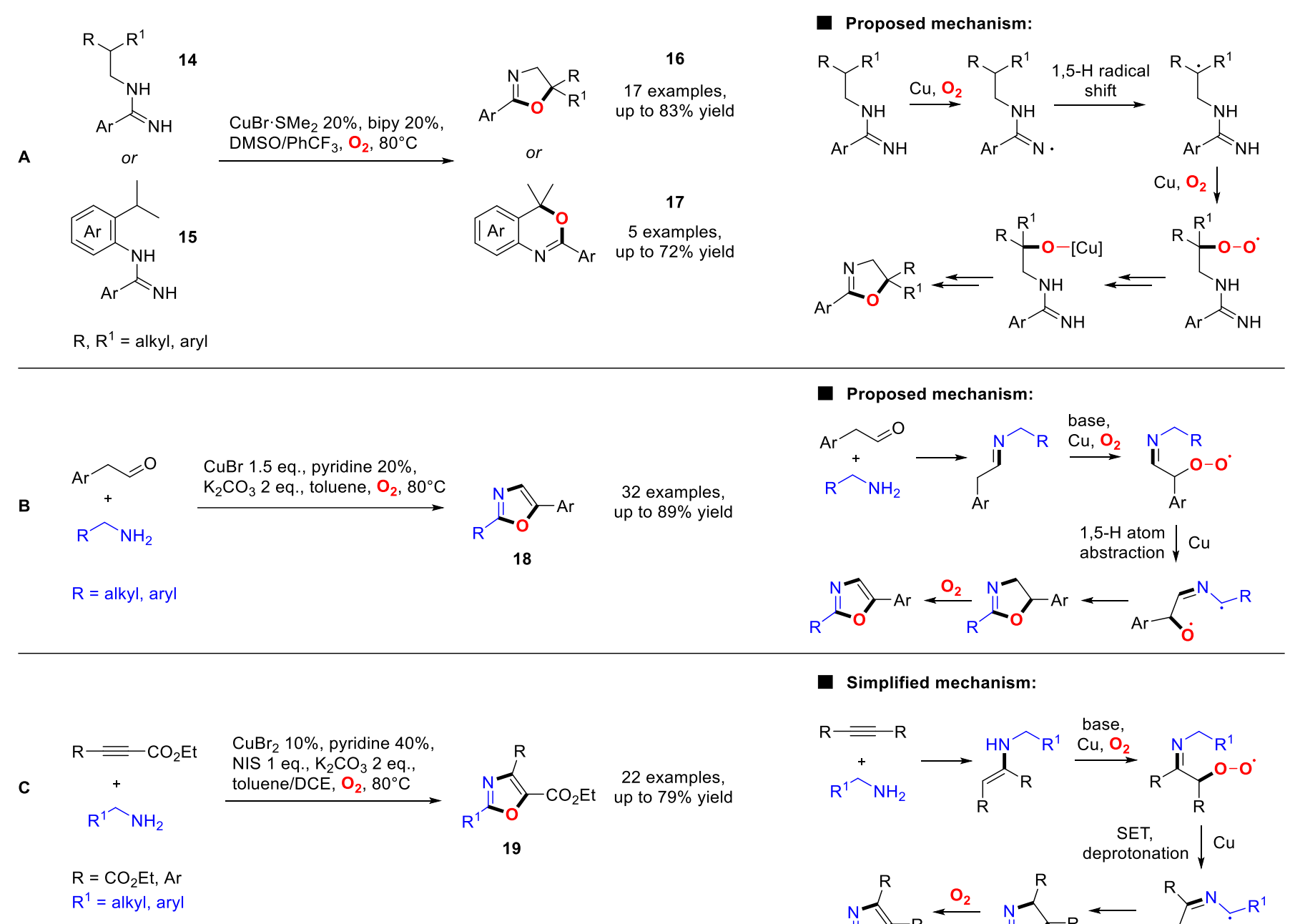

\section{- Simplified mechanism:}
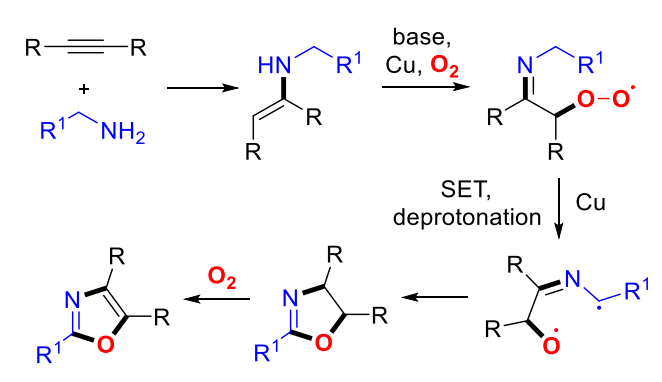

Scheme 4. Synthesis of aza-heterocycles via $\mathrm{Csp}^{3}-\mathrm{H}$ oxygenation / C-O bond formation.

\section{Oxidative Isocyanide Insertion}

Isocyanides have recently replaced the isoelectronic carbon monoxide as a one-carbon synthon in a variety of protocols, including for the synthesis of heterocycles, ${ }^{20-21}$ due to their lower toxicity and their easier handling. Similarly to the oxidative insertion of $\mathrm{CO}$ (see Section 4), an important strategy for the synthesis of a variety of heterocycles is the oxidative insertion of isocyanides into a metallacycle (usually Pd), followed by reductive elimination. Only the terminal carbon of the isocyanide is thus incorporated into the ring, and an aminosubstituted heterocycle is obtained. In this case the oxidant is necessary to restore the active $\mathrm{Pd}(\mathrm{II})$ species from $\operatorname{Pd}(0)$ after the reductive elimination. Non-oxidative versions of this reaction were reported before 2010, making use of organic halides as substrates and not requiring any oxidant (

Scheme 5, left). A number of different heterocyclic structures can be easily obtained using this method, by formation of either a C-C and a C-heteroatom bond, or two C-heteroatom bonds ( Scheme 5, right). These protocols are discussed in Sections 3.1 and 3.2. 
A different reactivity pathway has also been recently discovered. Here an initial isocyanide insertion is followed by an acyl migration, resulting in both the $\mathrm{C}$ and $\mathrm{N}$ atoms of the isocyanide being incorporated into the ring. This reactivity has very different implications and mechanism, and is treated in Section 3.3.
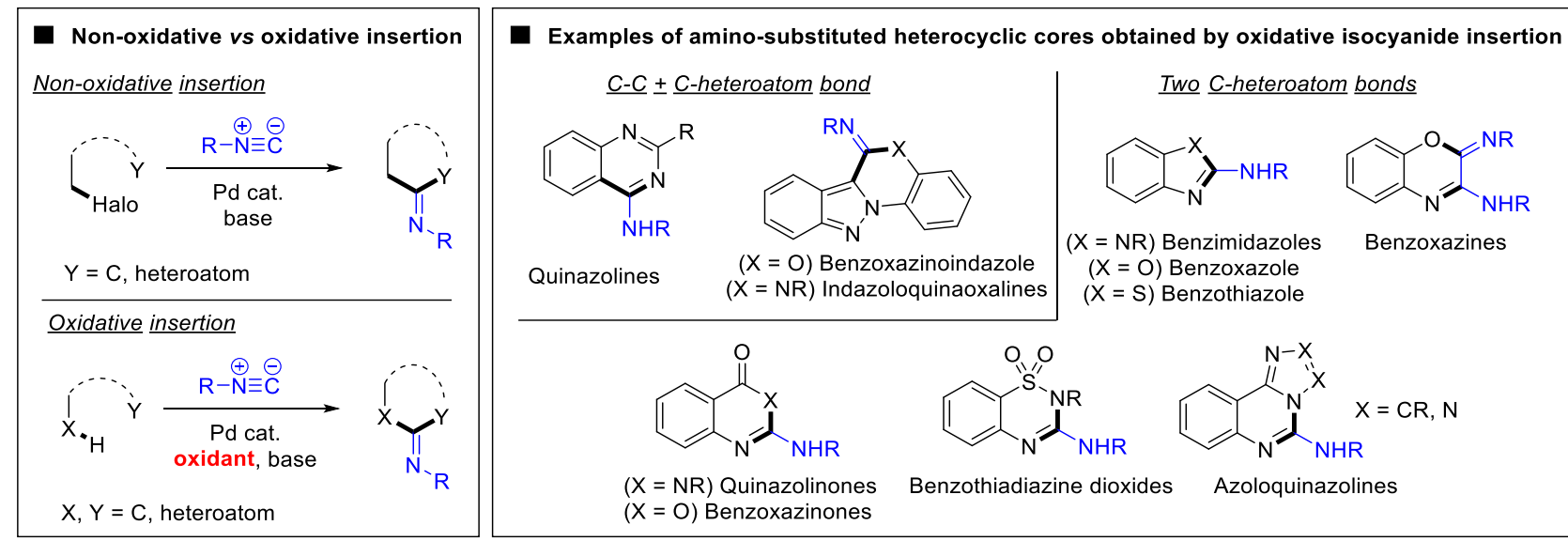

Scheme 5. Left: oxidative vs non-oxidative isocyanide insertion. Right: examples of synthetic applications.

\subsection{Formation of C-C + C-heteroatom bonds}

The first example of aerobic oxidative insertion of isocyanides into a heterocyclic ring was reported in 2011 by Zhu and co-workers for the synthesis of 4-aminoquinazolines $21 .^{22}$ The authors reported the reaction between $\mathrm{N}$-arylamidines $\mathbf{2 0}$ and isocyanides in the presence of $\mathrm{Pd}(\mathrm{OAc})_{2}$ under an $\mathrm{O}_{2}$ atmosphere, leading to the desired products in generally good yields (Scheme 6A). It is worth noting that the use of oxygen gave almost double yields of the desired product than other common oxidants, such as oxone and benzoquinone (BQ). The reaction worked well with both aliphatic and aromatic isocyanides, although stability issues somewhat hampered the use of the latter. As higher yields were obtained when electron-donating groups were present on the phenyl ring, the authors proposed an electrophilic aromatic substitution as the $\mathrm{Csp}^{2}-\mathrm{H}$ activation step (Scheme 6, right). ${ }^{22}$

An analogous mechanism was later suggested for the synthesis of benzoxazinoindazoles 23 and indazoloquinaoxalines $\mathbf{2 4}$ from indazole derivatives $22 .{ }^{23}$ While the use of oxygen was efficient for the former, the latter were obtained in higher yields employing $\mathrm{CuCl}_{2}$ as oxidant (Scheme 6B).

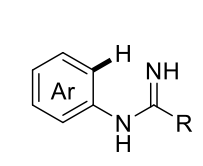

A

$$
\mathrm{R}=\mathrm{Ar}, \mathrm{tBu}, \mathrm{Cy}
$$
$\mathrm{R}^{1}=\mathrm{tBu}, \mathrm{iPr}, \mathrm{Cy}, \mathrm{Ar}$

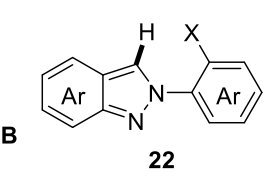

$\mathrm{R}=\mathrm{tBu}, \mathrm{Cy}$

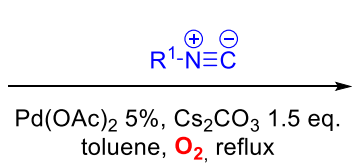
toluene, $\mathrm{O}_{2}$, reflux

21

30 examples, up to $97 \%$ yield
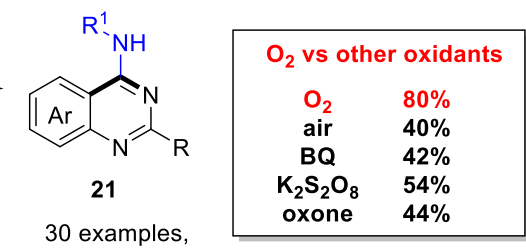

(n)

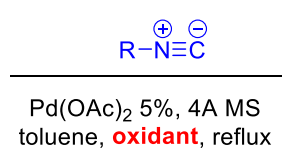

$23\left(X=O\right.$, oxidant $\left.=\mathrm{O}_{2}\right), 17$ examples, up to $98 \%$ yield $24\left(X=\mathrm{NH}\right.$, oxidant $\left.=\mathrm{CuCl}_{2} \cdot 2 \mathrm{H}_{2} \mathrm{O}\right), 8$ examples, up to $78 \%$ yield

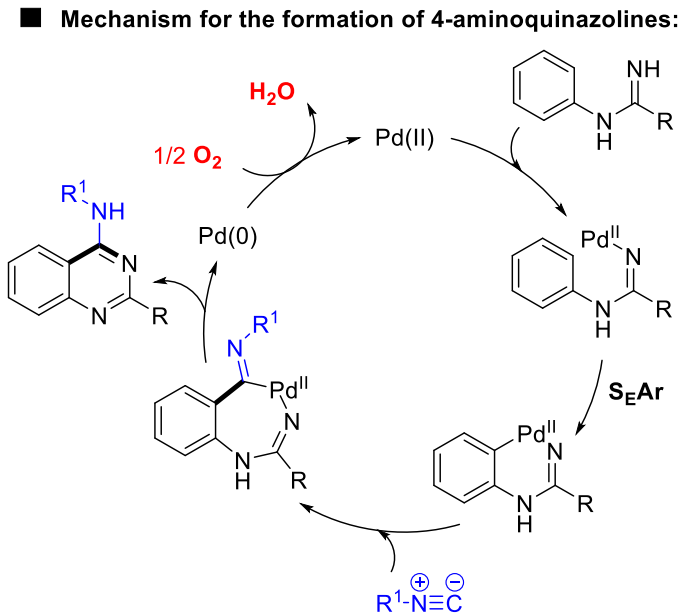

Scheme 6. Oxidative isocyanide insertion leading to $C-C+C$-heteroatom bond formation. 


\subsection{Formation of two C-heteroatom bonds}

The use of $\mathrm{O}_{2}$ as oxidant for isocyanide insertions between two heteroatoms was first reported by Maes/Orru/Ruijter in 2012 for the synthesis of 2-aminobenzimidazoles 25, and other heterocyclic cores were synthesized under similar reaction conditions (26-31, Scheme 7A). ${ }^{24}$ A notable aspect is the variety of
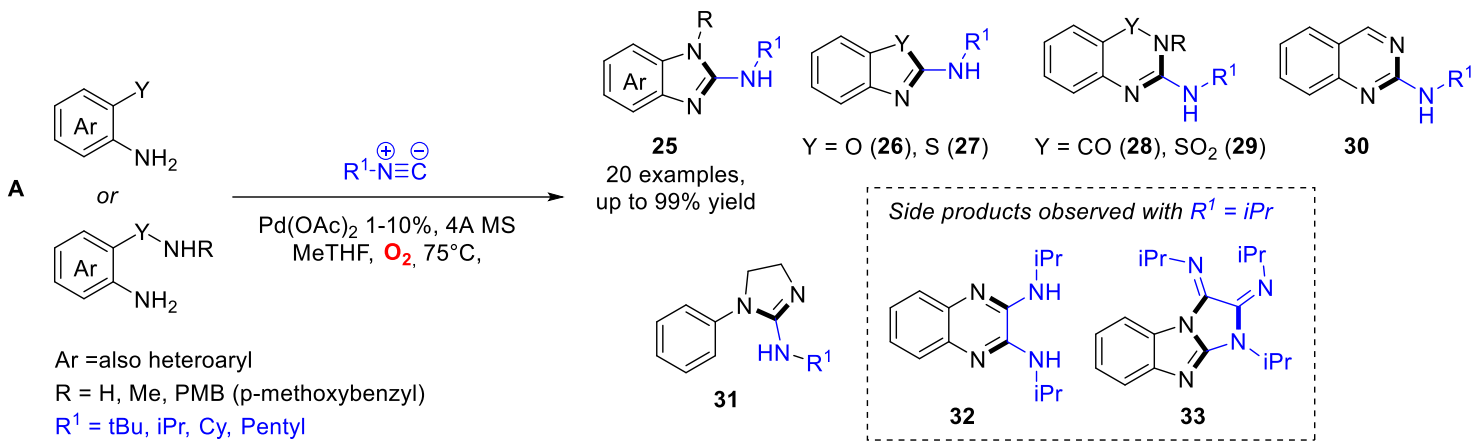

$\mathrm{R}^{1}=\mathrm{tBu}, \mathrm{iPr}, \mathrm{Cy}$, Penty

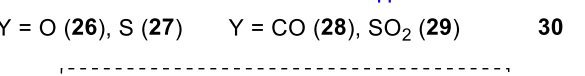

Side products observed with $R^{1}=i \operatorname{Pr}$
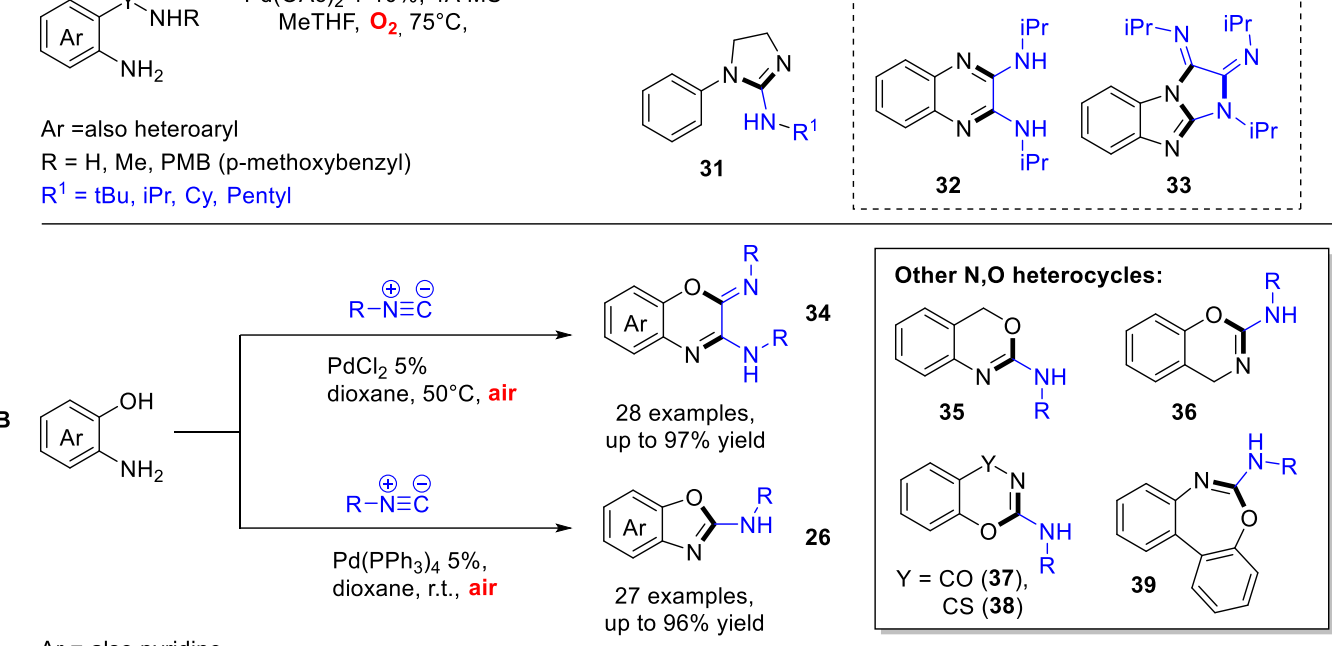

$\mathrm{Ar}=$ also pyridine

$\mathrm{R}=\mathrm{tBu}, \mathrm{nBu}, \mathrm{Cy}, \mathrm{Bn}, \mathrm{CH}_{2} \mathrm{COOEt}, 1,1,3,3$ - tetramethylbutyl, 2-morpholinoethyl, 4-OMe-Ph, 2,6-dimethylphenyl

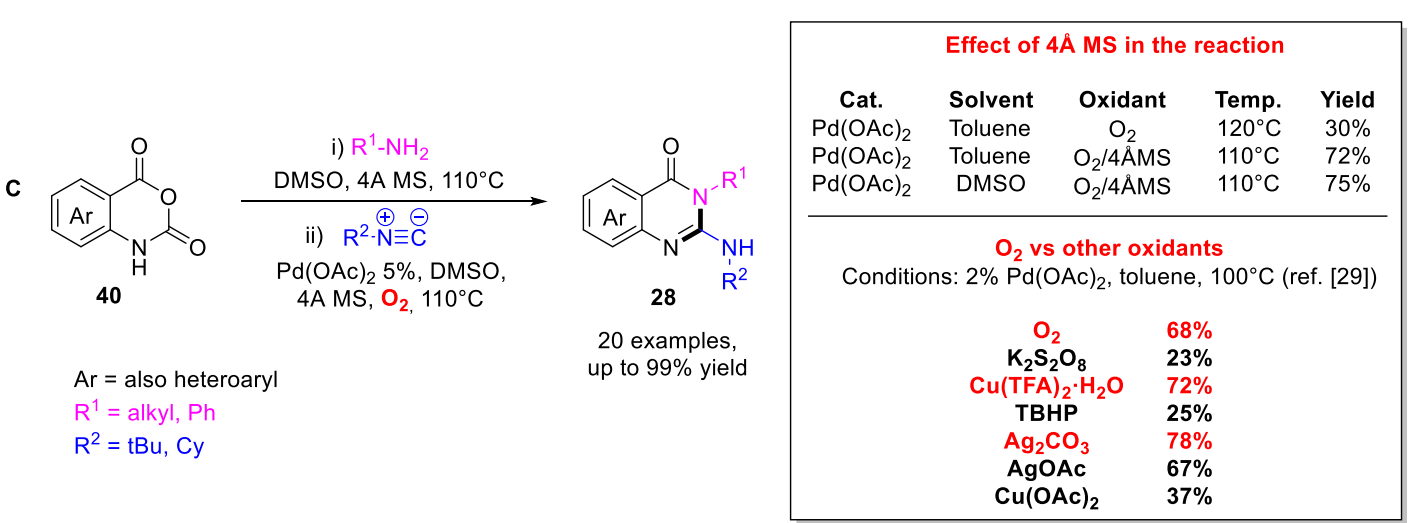

Mechanism for the formation of 2-aminobenzimidazole:

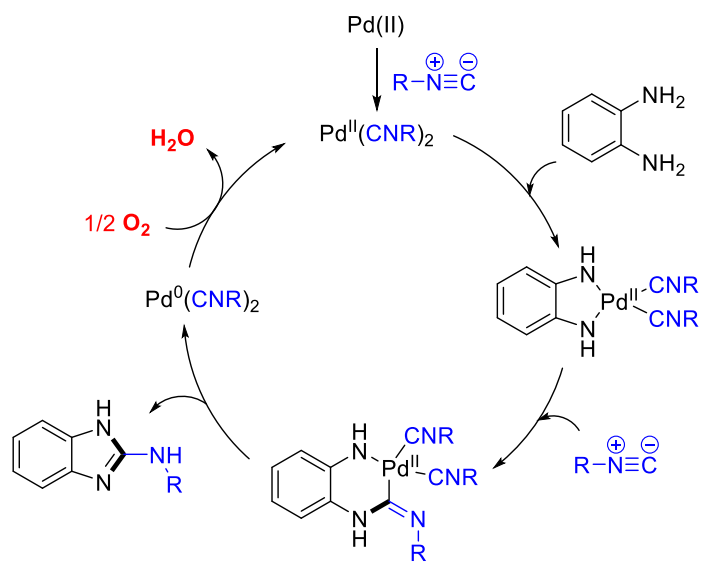

Scheme 7. Oxidative isocyanide insertion leading to C-heteroatom + C-heteroatom bond formation. 
(hetero)aromatic precursors tolerated in the synthesis of 2-aminobenzimidazole derivatives. Again high yields were obtained from electron-rich starting materials (substituent on $\mathrm{Ar}$ ) using only $1 \% \mathrm{Pd}$, while strong electronwithdrawing groups required the use of higher catalyst loadings. Concerning the scope in isocyanides, a few aliphatic substituents were tolerated such as $t \mathrm{Bu}$, cyclohexyl, pentyl and $i \operatorname{Pr}$. The use of $i \mathrm{PrNC}$ as reactant resulted in certain amounts of side-products 32-33, derived from the insertion of two and three equivalents of isocyanides. $^{24}$

A similar insertion of two molecules of isocyanide was also reported by Jiang and co-workers. In this case, a change in catalyst and reaction temperature proved crucial to selectively obtain the mono- or di-insertion product (34 and 26, Scheme 7B). ${ }^{25}$ A large variety of isocyanides was suitable for both transformations, including functionalized aliphatic, benzylic and aromatic isocyanides. Moreover, several other N,O heterocycles, including dibenzoxazepines 39, were prepared using similar conditions.

Several other reports on the synthesis of related heterocycles were recently published, ${ }^{26-27}$ and an original decarboxylative variation was reported by Sharada in 2015. Here the authors employed isatoic anhydrides $\mathbf{4 0}$ as starting materials, which undergo decarboxylation-amination and isocyanide insertion in a one-pot fashion, to provide 2-amino-4-quinazolinones $\mathbf{2 8}$ (Scheme 7C). ${ }^{28}$ The use of molecular sieves was essential for obtaining good yields. The same transformation was achieved using $\mathrm{Ag}_{2} \mathrm{CO}_{3}$ as oxidant by Cai. ${ }^{29} \mathrm{~A}$ comparison of the results obtained for this reaction with different oxidants is reported in Scheme 7C. Although higher yields were obtained with $\mathrm{Ag}$ or $\mathrm{Cu}$ oxidants rather than $\mathrm{O}_{2}$, the use of two equivalents of the metal makes these options considerably more wasteful and more expensive.

The proposed mechanism for the isocyanide insertion between two heteroatoms (Scheme 7, bottom) ${ }^{24}$ is only slightly different from the one shown in Scheme 6, however other mechanisms have been suggested to account for the formation of deamination side-products, sometimes observed in small amounts. ${ }^{25}$

\subsection{Isocyanide insertion / acyl migration}

In 2014, while designing a catalytic system able to tolerate strongly coordinating heterocyclic substituents in group-directed $\mathrm{C}-\mathrm{H}$ functionalization, ${ }^{30} \mathrm{Yu}$ et al. discovered a new reactivity pattern in isocyanide insertion. Subjecting $\mathrm{N}$-methoxy amides $\mathbf{4 1}$ to $\mathrm{Pd}$-catalyzed aerobic isocyanide insertion, instead of the expected aminosubstituted heterocycles, compounds $\mathbf{4 2}$ were isolated in high yields (

Scheme 8). ${ }^{31}$ These are formed by an initial isocyanide insertion, followed by a 1,3 acyl migration process, resulting in the incorporation of both the $\mathrm{C}$ and $\mathrm{N}$ atoms into the ring. Counter-intuitively, the reaction performed much better under air than under $\mathrm{O}_{2}$. This was later proved to be due to the faster oxidation of the isocyanide to isocyanate under $\mathrm{O}_{2}$, and the concomitant inhibition of the insertion reaction. ${ }^{32}$ Detailed mechanistic investigation of this process has been undertaken by Stahl ${ }^{32}$ and Wang. ${ }^{33}$ 


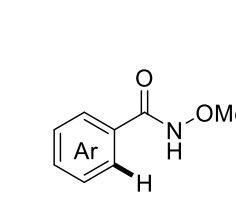

or

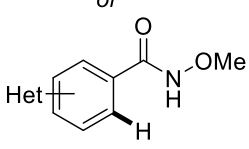

41

$\mathrm{Ar}=$ also heteroaryl $\mathrm{Het}=$ heterocycle

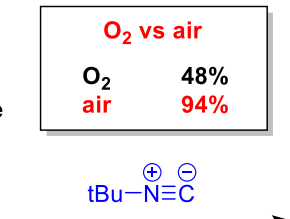

$\mathrm{Pd}(\mathrm{dba})_{2} 2.5 \%$ dioxane, $80^{\circ} \mathrm{C}$, air

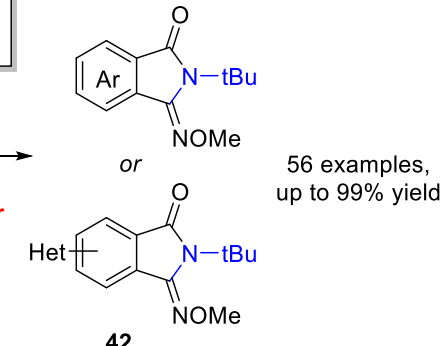

42

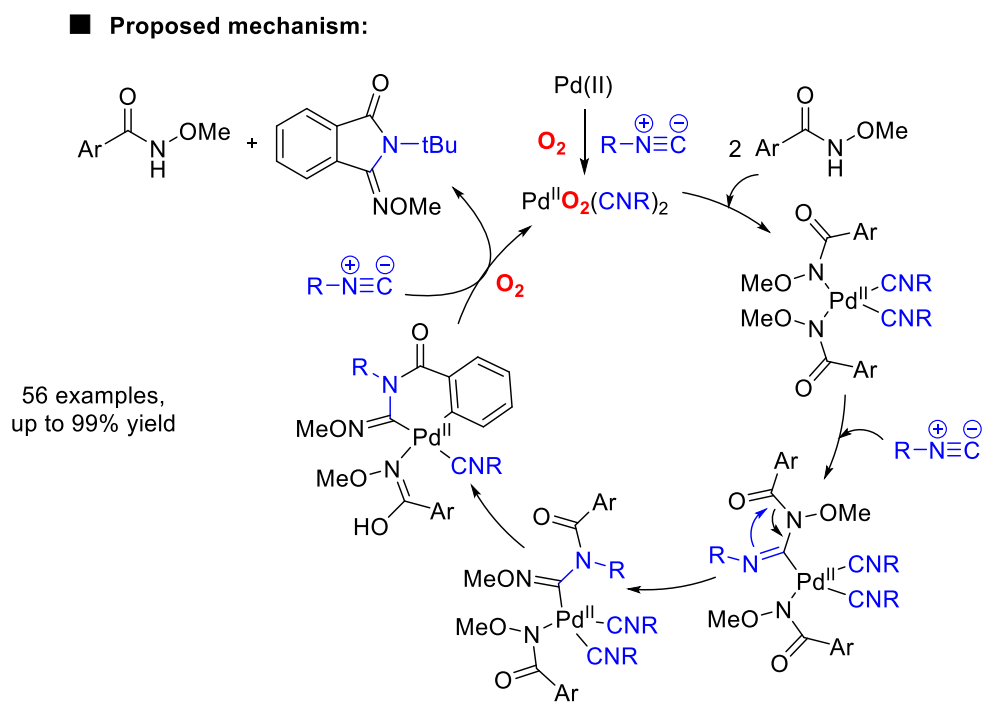

Scheme 8. Synthesis of isoindolinone derivatives $\mathbf{4 2}$ via oxidative isocyanide insertion / acyl migration.

\section{Oxidative $\mathrm{CO}$ Insertion}

As mentioned in the previous section, oxidative carbonylations catalyzed by transition metals have been explored even before the use of related isocyanides. As these transformations have already been reviewed, ${ }^{34-35}$ and generally follow the same mechanism as described for isocyanides, these will not be treated in depth here. Nonetheless, a few recent applications have appeared in the literature that are worth mentioning. As the mixture $\mathrm{CO} / \mathrm{O}_{2}$ can generate considerable safety hazard, particular attention must be given to the reaction conditions (in particular pressure and ratio of the two gases), to avoid explosive mixtures.

While CO insertions are typically catalyzed by Pd, Kaneda and co-workers disclosed the use of hydrotalcitesupported Au nanoparticles (Au@HT) as a heterogeneous catalytic system for the carbonylation of aminophenols, leading to benzoxazolinones and benzimidazolones 43-44 (

Scheme 9A). ${ }^{36}$ The solid catalyst could be recycled and re-used for a few runs without a reduction in yield, and no leaching was detected from the heterogeneous system.

Interesting cascade reactions were reported under Pd catalysis by Gabriele/Mancuso/Della Ca' for the synthesis of 8-membered lactams $\mathbf{4 6}$ and fused furo[3,4-b]indol-1-ones $\mathbf{4 8}$ (

Scheme 9B-C). ${ }^{37-38}$ The same authors also reported the Pd-catalyzed carbonylation of aminoalcohols/phenols in an ionic liquid (

Scheme 9D). ${ }^{39}$ Notably, after separation of the products, the ionic liquid medium (with the catalyst) could be recycled several times.

A continuous-flow version of this reaction was recently reported by Kappe (

Scheme $9 \mathrm{E}) .{ }^{40}$ While in batch the use of a large excess of $\mathrm{CO}$ or the use of air instead of pure $\mathrm{O}_{2}$ is required to provide a certain operational safety, the use of flow allowed the use of equal pressures (and lower excesses than in batch) of $\mathrm{CO}$ and pure $\mathrm{O}_{2}$ in a safe manner. A range of 5- and 6-membered heterocycles were synthesized with this flow process. 

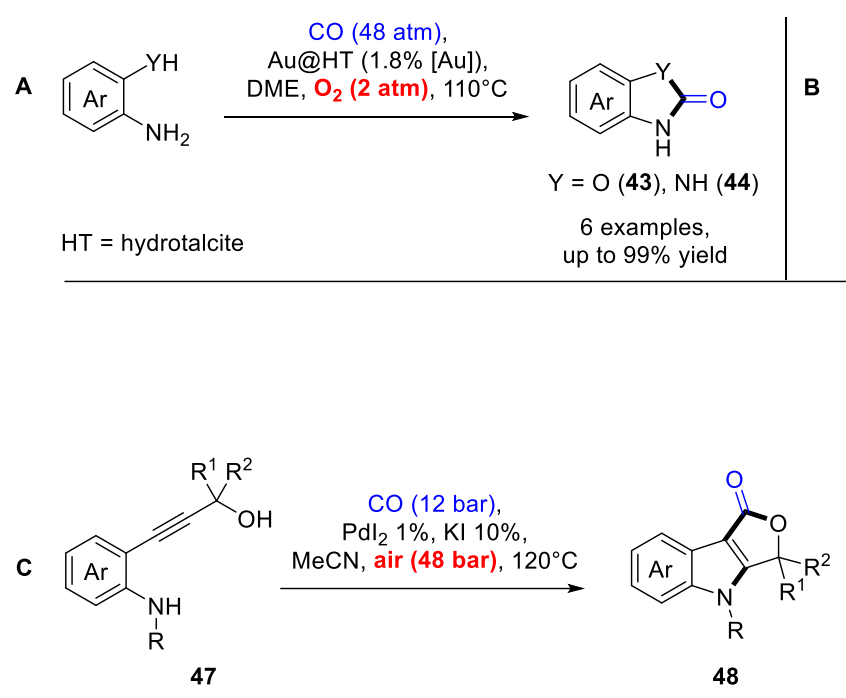

$\mathrm{R}=\mathrm{H}$, alkyl

$R^{1}, R^{2}=H$, alkyl, $P h$

19 examples, up to $98 \%$ yield
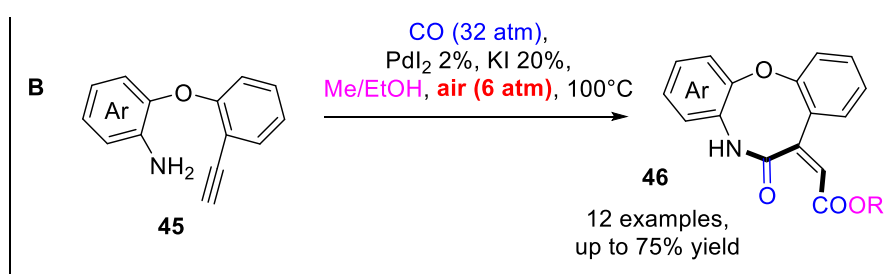

Proposed mechanism:

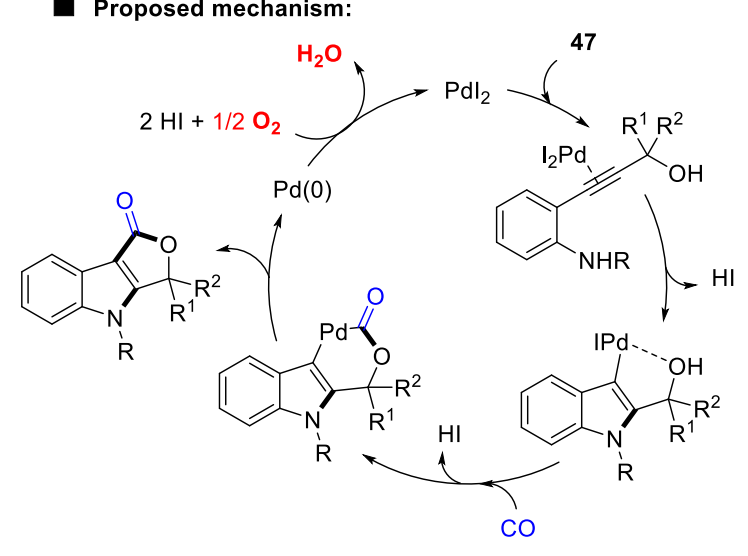

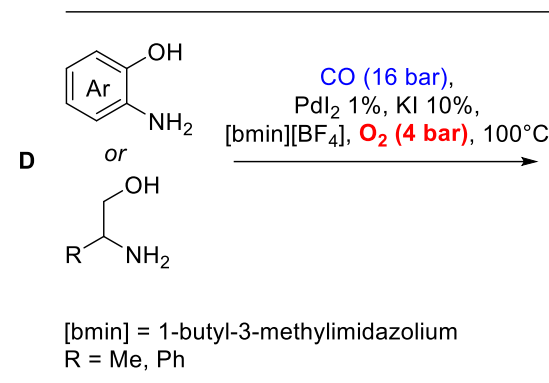
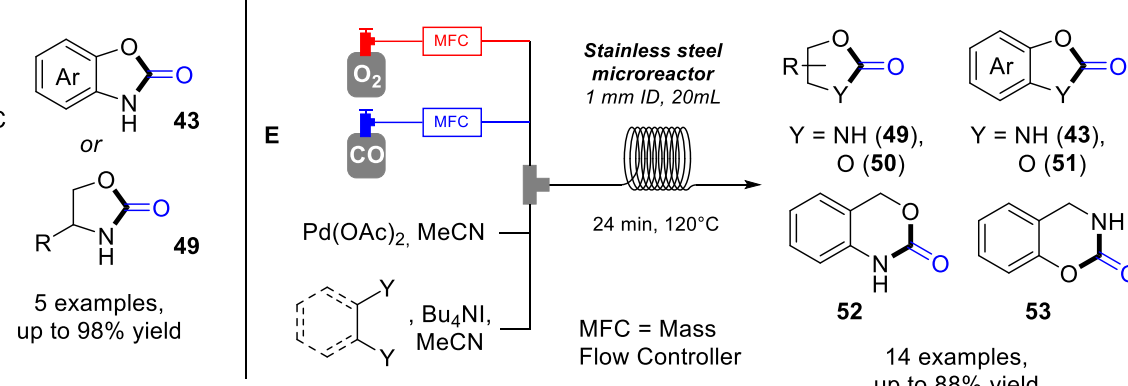

$\mathrm{Y}=\mathrm{NH}(\mathbf{4 3})$, $\mathrm{O}(51)$

52

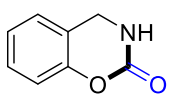

14 examples, up to $88 \%$ yield

Scheme 9. Synthesis of heterocycles via oxidative carbonyl insertion.

\section{Oxidative Friedlander Annulation - Acridine Synthesis}

The Friedlander annulation of 2-acyl or 2-formylanilines with ketones for the synthesis of quinolines has been known for over a century. ${ }^{41-42}$ An interesting application of this chemistry in combination with aerobic oxidation has been recently explored for the formation of acridine cores. The role of oxygen here is limited to the dehydrogenation of the aliphatic ring introduced with the Friedlander reaction, and therefore not strictly involved in the synthesis of the heterocycle itself. Despite not being a general, common strategy for the synthesis of heterocycles, the importance of the acridine core and the lack of other general aerobic oxidation strategies for the synthesis of this specific moiety justify the addition of a small section in this review.

Wang first reported in 2016 a metal-free method employing both TBHP and $\mathrm{O}_{2}$ as oxidants, under acidic conditions (54,

Scheme 10). ${ }^{43}$ The same protocol could be applied for the synthesis of other heterocycles, employing 3amino-2-acylbenzofuranes (55). Basing their studies on this report, Wu and Deng developed analogous processes under Pd catalysis, which avoided the use of TBHP (

Scheme 10). ${ }^{44-45}$ The postulated mechanism for this process involves an acid-catalyzed dehydrative coupling of 2-acylanilines and cyclohexanone (Friedlander annulation), followed by dehydrogenation, leading to the 
desired acridine core. This mechanism is supported by the formation of quinolines 56 when using nonaromatizable ketones, ${ }^{44}$ and by the isolation of the oxygenated intermediate $57 .{ }^{43}$

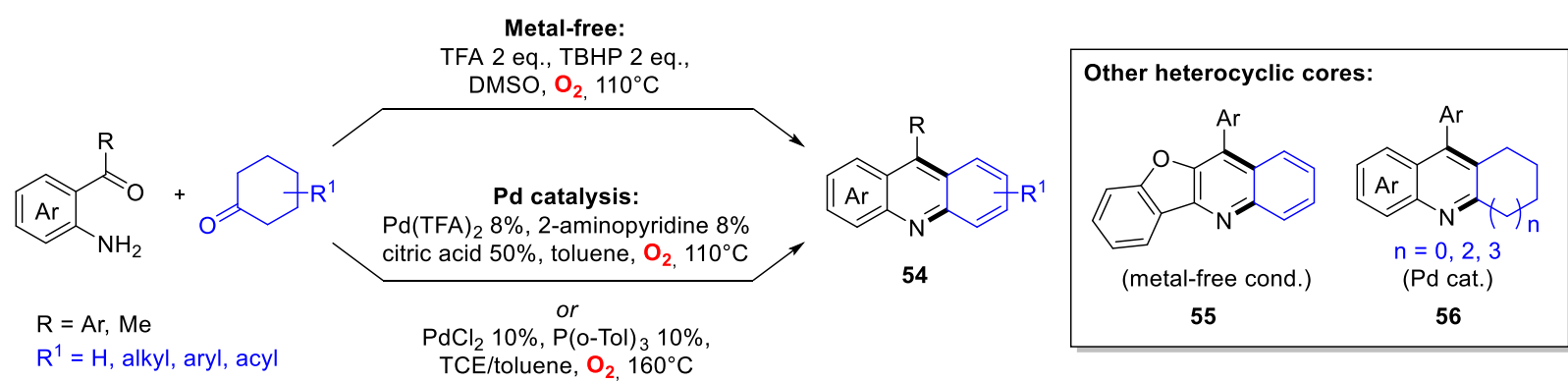

- Simplified mechanism:
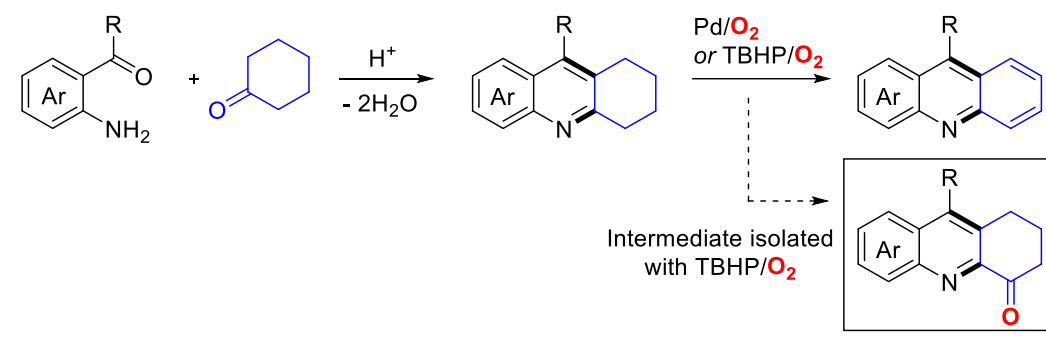

Scheme 10. Aerobic oxidative synthesis of acridine cores via Friedlander annulation.

\section{Oxidative N-Heteroatom Bond Formation}

A variety of 1,2-diheterocycles and related triheterocycles can be easily obtained via aerobic oxidative $\mathrm{N}$ heteroatom bond formation, several examples of which have recently appeared in the literature. Oxidative $\mathrm{N}$ $\mathrm{N}, \mathrm{N}-\mathrm{O}, \mathrm{N}-\mathrm{S}$ and $\mathrm{N}-\mathrm{Se}$ bond formation have all been investigated for the synthesis of a range of 4- to 6-membered heterocyclic moieties.

\subsection{Oxidative $\mathrm{N}-\mathrm{N}$ bond formation}

Oxidative $\mathrm{N}-\mathrm{N}$ bond formation is not a major research field in organic synthesis, but has been reported in the past to occur using strong oxidants or stoichiometric/superstoichiometric amounts of $\mathrm{Cu} .{ }^{46-47}$ Since the 2000s, several reports have appeared on the use of catalytic $\mathrm{Cu} / \mathrm{O}_{2}$ combination as a greener alternative. These methods can be exploited for the synthesis of pyrazoles, indazoles, or triazoles, which are traditionally synthesized using reagents already containing a N-N bond (i.e. hydrazines).

Several variations for the synthesis of such heterocycles have been reported with the $\mathrm{Cu} / \mathrm{O}_{2}$ methodology, often in a cascade or one-pot fashion. In such cases, an initial intermolecular reaction (typically non-oxidative) provides a suitable coordinating $\mathrm{N}, \mathrm{N}$ substrate, which, upon coordination with $\mathrm{Cu}$, can be oxidatively coupled by a reductive elimination-like process. The role of oxygen is presumably the reoxidation of the resulting reduced $\mathrm{Cu}$ species, allowing catalyst turnover (oxidase-type). While the second step (oxidative $\mathrm{N}-\mathrm{N}$ bond formation) seems to be fairly easy under these conditions, the overall outcome of the reaction can be very dependent on the starting materials used and the coupling method. 
6.1.1 Synthesis of pyrazoles and indazoles. Pyrazoles are typically synthesized by reaction of hydrazines, hydrazones or analogues with alkynes, diols/diketones, or $\alpha, \beta$-unsaturated carbonyls. ${ }^{48} \mathrm{~A}$ variety of different pathways and synthons can be exploited for a N-N bond-forming process. In 2010 Glorius disclosed the serendipitous formation of pyrazoles upon treatment of enaminones $\mathbf{5 8}$ with $\mathrm{Cu}(\mathrm{OAc})_{2}$ in acetonitrile. ${ }^{46}$ Although this reaction required 1.5 eq. of $\mathrm{Cu}$ and was actually sensitive to $\mathrm{O}_{2}$ atmosphere, the protocol was later improved to employ only catalytic amounts of $\mathrm{Cu}(10 \%)$ and $\mathrm{O}_{2}$ as oxidant. ${ }^{49}$ This reaction worked efficiently with a range of substituted enaminones and nitriles, either aliphatic or aromatic, proving a versatile method for the synthesis of 1,3,4,5-tetrasubstituted pyrazoles 59 (

Scheme 11A). Nonetheless, the reaction outcome in aerobic conditions varied considerably depending on the substrate and the nitrile used, and the yields obtained were comparable or lower than with stoichiometric Cu.

Another important pyrazole synthesis was published by Jiang in 2014, occurring via a Cu-catalyzed multicomponent reaction between oximes, anilines and formaldehyde, furnishing 1,3-disubstituted (mostly diaryl) pyrazoles 61 (

Scheme 11B). ${ }^{50}$ The reaction under $\mathrm{N}_{2}$ gave good yields of the corresponding pyrazoline 62 , suggesting $\mathrm{O}_{2}$ to be only involved in the dehydrogenation of the ring, rather than the $\mathrm{N}-\mathrm{N}$ bond formation itself. This process is here likely promoted by the oxime moiety, which acts as an internal oxidant. A similar approach was independently described by Cui and co-workers. In this case, the cyclization of tosylimines and oxime esters also resulted in pyrazolines, but also required superstoichiometric Cu salts. ${ }^{51}$ Although the reaction was performed under air, aerobic oxygen was probably not essential for the process, due to the high amounts of $\mathrm{Cu}$ used.

For the synthesis of indazoles 63, the Glorius group reported a tandem process involving Rh-catalyzed groupdirected ${ }^{30} \mathrm{C}-\mathrm{H}$ amidation (intermediate 64) and subsequent $\mathrm{Cu}$-catalyzed aerobic $\mathrm{N}-\mathrm{N}$ bond formation (

Scheme 11C).52 An analogous cascade process, only employing $\mathrm{Cu}$ as catalyst for both the directed $\mathrm{C}-\mathrm{H}$ sulfonamidation and the N-N coupling was reported in 2014 by Zhu, but under inert conditions (the azide was suggested to act as both coupling partner and oxidant). ${ }^{53}$

Another indazole synthesis was reported by Chen and Tang in 2016 starting from ortho-aminobenzonitriles 1

Scheme 11D). ${ }^{54}$ Ketimines 65 were prepared from the nitriles via reaction with a Grignard or organolithium reagent, and subsequent Cu-catalyzed oxidation resulted in the desired indazoles 66. As a range of organometallic species and $\mathrm{N}$-substituents could be tolerated under these conditions, this method appears complementary to Glorius's procedure, and several compounds were prepared in a multigram scale. ${ }^{54}$ When a $\mathrm{N}$-aryl substituted substrate was employed, the aerobic oxidation also resulted in a cascade ortho hydroxylation of the $\mathrm{N}$-aryl ring (67). ${ }^{55}$ Also in this instance the reaction proved robust and good-yielding even at gram scale. It is noteworthy that the use of indazole as a pre-formed directing group was inefficient for the hydroxylation, as well as the amino group alone..$^{55}$ 


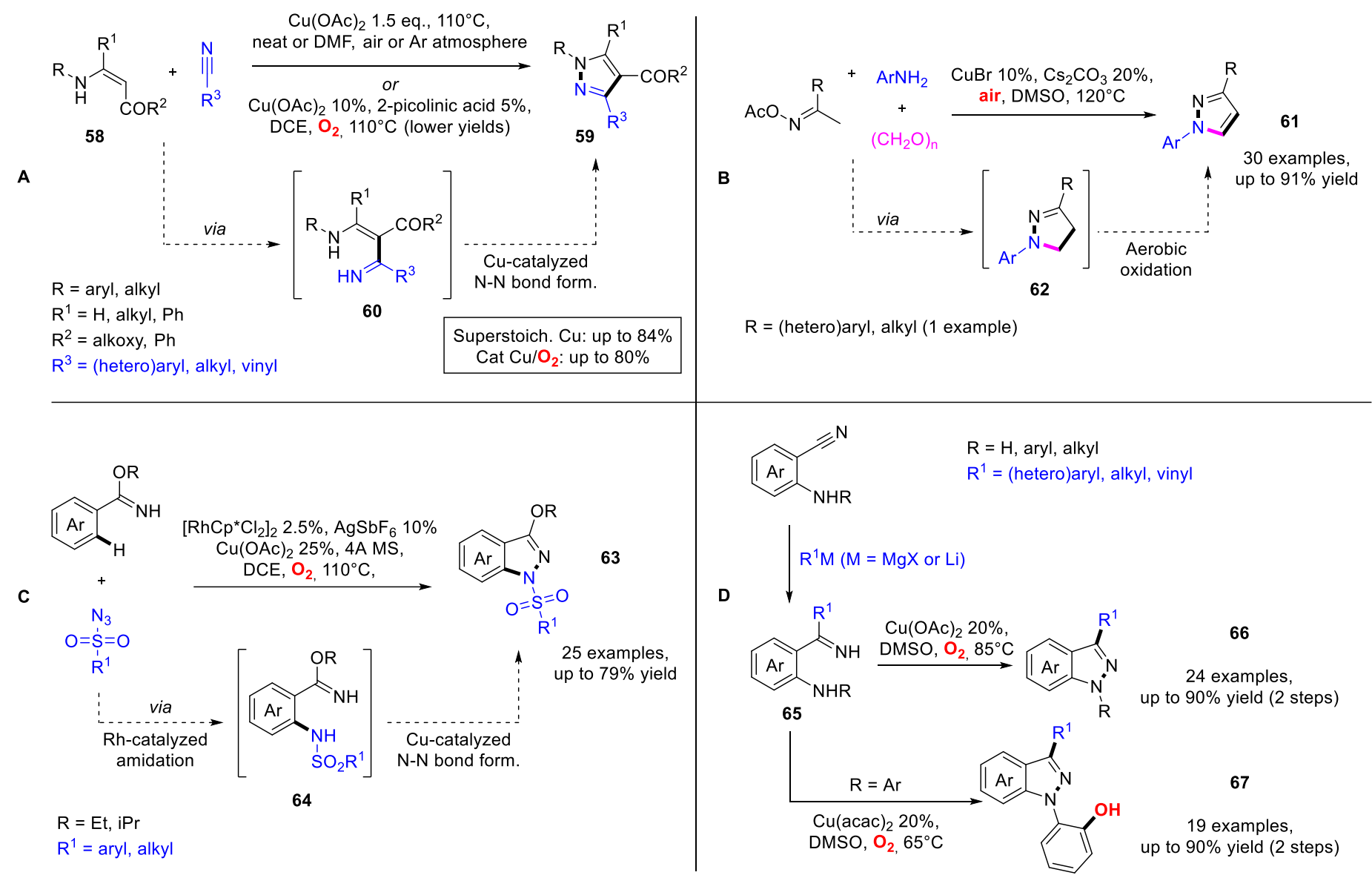

Scheme 11. Synthesis of pyrazole and indazole derivatives via oxidative N-N bond formation.

6.1.2. Synthesis of triazoles. 1,2,4-Triazoles are easily formed by addition of amidines to nitriles (or analogous reactants), followed by intramolecular oxidative N-N coupling. In 2009 this strategy was employed by Nagasawa for the synthesis of triazolopyridines 68 and triazoles 69 in generally good yields (

Scheme 12A). ${ }^{56}$ This cascade protocol worked efficiently with a variety of aromatic nitriles, but yields were limited when using cyanamides, likely due to difficult addition to the nitrile in such compounds. ${ }^{57}$ Given the interest in the 2-amino-[1,2,4]triazolo[1,5-a]pyridine scaffold in medicinal chemistry, researchers at Roche modified Nagasawa's protocol employing guanidinic precursors 71 (

Scheme 12B). ${ }^{57}$ This method allowed the efficient synthesis of 2-aminotriazolopyridines 72 and analogues 7376 from diazaheterocyclic guanidines. Another very similar procedure to Nagasawa's was reported by Zhang, this time applied to the synthesis of trisubstituted triazoles 77. Importantly, the authors also reported the synthesis of disubstituted triazoles 69 using aliphatic nitriles (1 example only), typically not reactive under these conditions (

Scheme 12C). ${ }^{58}$

The synthesis of 1,2,4-trazoles 69 by condensation or dimerization of amidines or imidates (both derived from nitriles) was reported by $\mathrm{Fu}^{59}$ and Beifuss ${ }^{60}$ (

Scheme 12D-E). These processes result in the same intermediate obtained from the addition of amidines to nitriles (70), but the long reaction time, the tendency of aromatic amidines to dimerize, and/or the complex reaction mixture employed make these methods not highly efficient, despite synthetically useful yields of 60- 
$70 \%$ could be achieved, depending on the substituents. Limited yields were also obtained using an anaerobic protocol starting from nitriles/hydroxylamine combinations. ${ }^{61}$
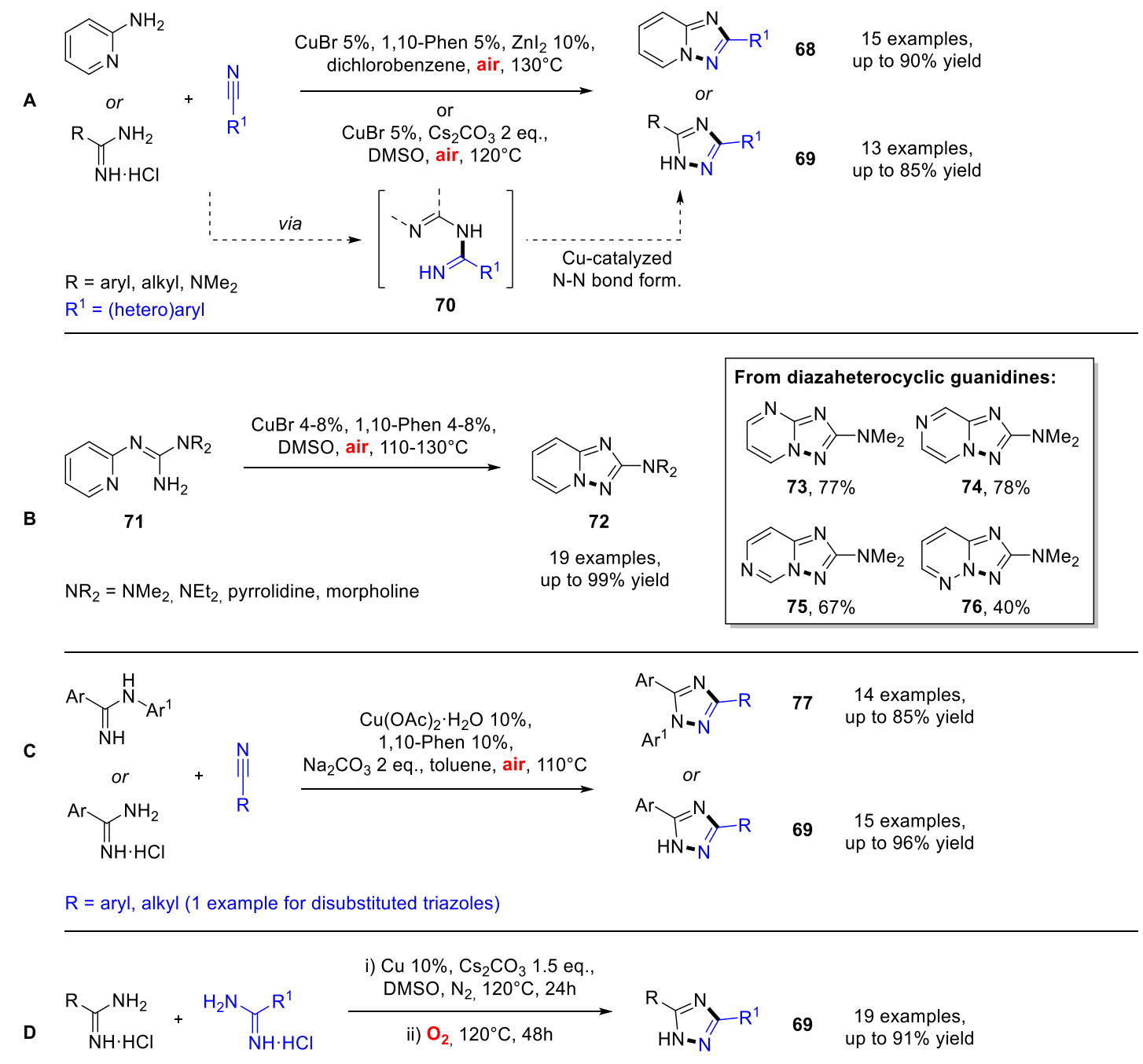

$\mathrm{R}=$ (hetero)aryl, alkyl

$\mathrm{R}^{1}=$ (hetero)aryl

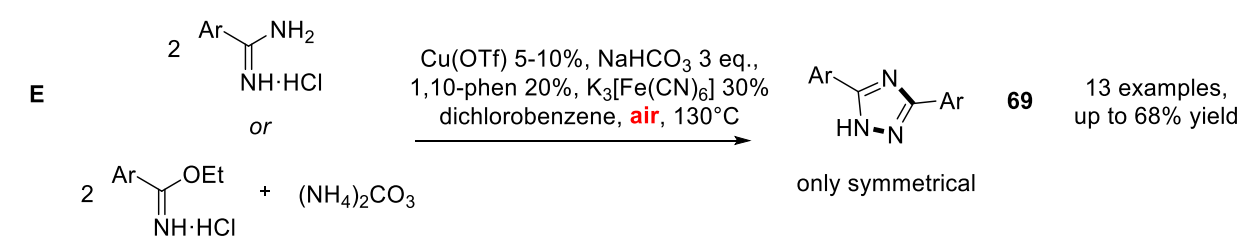

Scheme 12. Synthesis of 1,2,4-triazole derivatives via oxidative N-N bond formation.

The synthesis of 1,2,3-triazoles can be accomplished via Cu-catalyzed intramolecular coupling between a hydrazine and another $\mathrm{N}$ moiety in the substrate, as described by Nagasawa for the synthesis of 1,2,3triazolopyridines 80 (

Scheme 13A). ${ }^{62}$ The reaction could be performed either in one step starting from 2-acylpyridine hydrazones $\mathbf{7 8}$, or in a one-pot manner starting from the corresponding 2-acylpyridines $\mathbf{7 9}$. High yields were obtained with 
both protocols. Mechanistic studies suggested that the re-oxidation of the $\mathrm{Cu}(\mathrm{I})$ species formed during the reaction was the rate-determining step, and that the acetate anion acted as both a ligand and a base to deprotonate the hydrazone. ${ }^{62}$

An interesting protocol for the synthesis of 2,4,5-triaryl 1,2,3-triazoles 82 was disclosed by Punniyamurthy, via dimerization of bisarylhydrazones $\mathbf{8 1}$ (

Scheme 13B). In this process the $\mathrm{Cu}$ catalyst is also involved in the initial C-C bond formation. ${ }^{63}$

Although one example of asymmetric 1,2,3-triazole was shown, the above method is clearly more suitable for the synthesis of symmetric products. A complementary method, starting from hydrazonoketones 84 and an ammonium source, which allows the synthesis of variedly functionalized triazoles, was reported by Lin in 2015 (

Scheme 13C) ${ }^{64}$ With this procedure the C4 and C5 positions of the triazole can be functionalized with a variety of carbonyls (esters, ketones, amides), aryl, or alkyl groups (85), although the yields are strongly dependent on the substitution pattern.
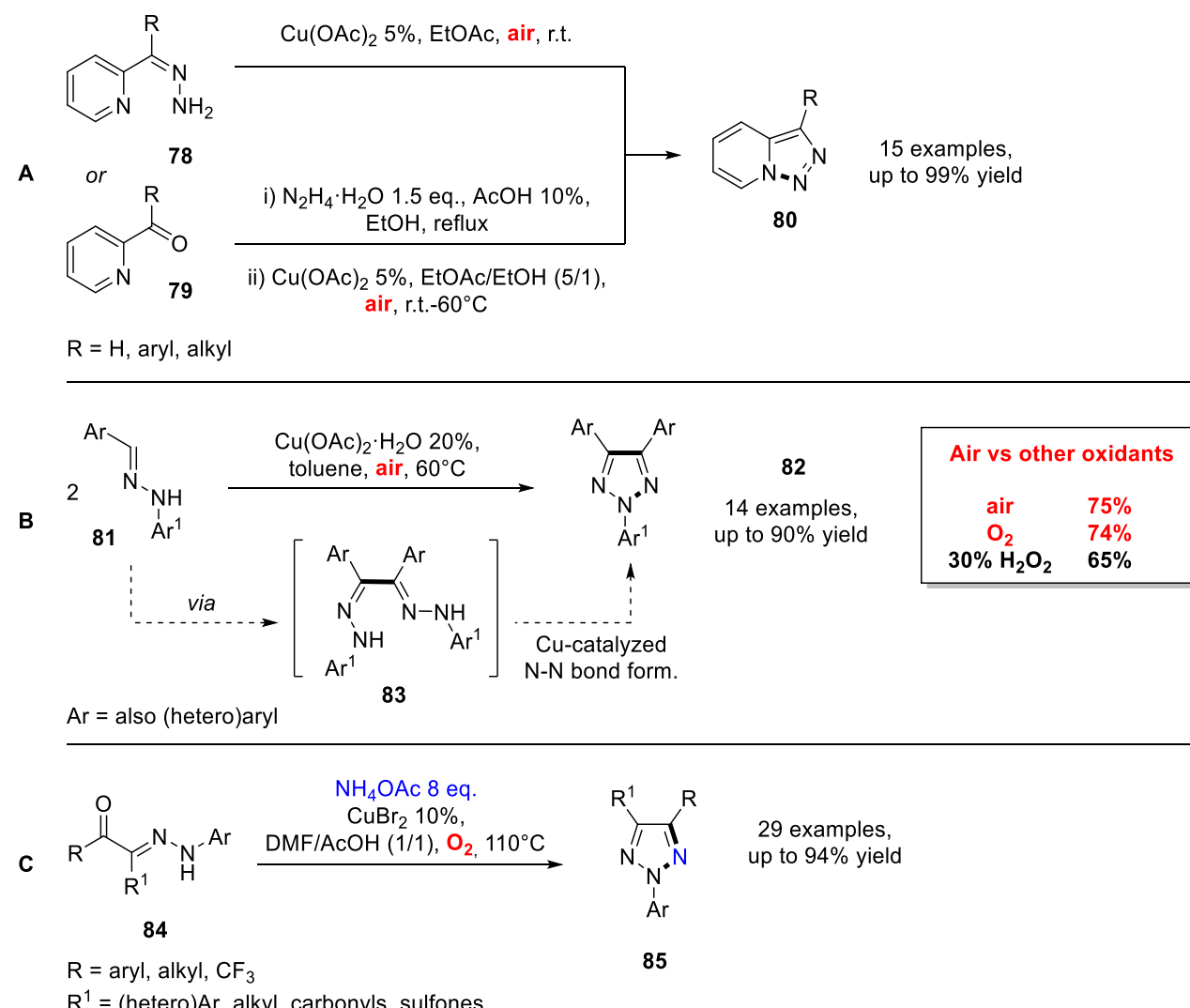

Scheme 13. Synthesis of 1,2,3-triazole derivatives via oxidative N-N bond formation.

\subsection{Oxidative $\mathrm{N}-\mathrm{O}, \mathrm{N}-\mathrm{S}$, and $\mathrm{N}-\mathrm{Se}$ bond formation}

Driven by the research on oxidative $\mathrm{N}-\mathrm{N}$ bond-forming processes, the formation of other heteroatomheteroatom bonds started to be investigated under similar catalytic conditions. Analogously to 1,2,4-triazoles, 1,2,4-oxadiazoles 86 could be prepared by addition of amides to nitriles, followed by oxidative N-O coupling, as described by Hong and co-workers ( 
Scheme 14A). ${ }^{65}$ The use of molecular sieves and $\mathrm{Znl}_{2}$, together with an excess of nitrile, were crucial for an efficient coupling/cyclization. Despite with variable yields (20-94\%), the reaction shows a broad scope in nitriles, in particular heteroaromatic and aliphatic. A couple of medicinally relevant compounds (a precursor of Ataluren, a drug against cystic fibrosis, and an inhibitor of penicillin-binding protein PBP2a, 87 and 88) could also be synthesized in decent yields, highlighting the synthetic utility of the method.
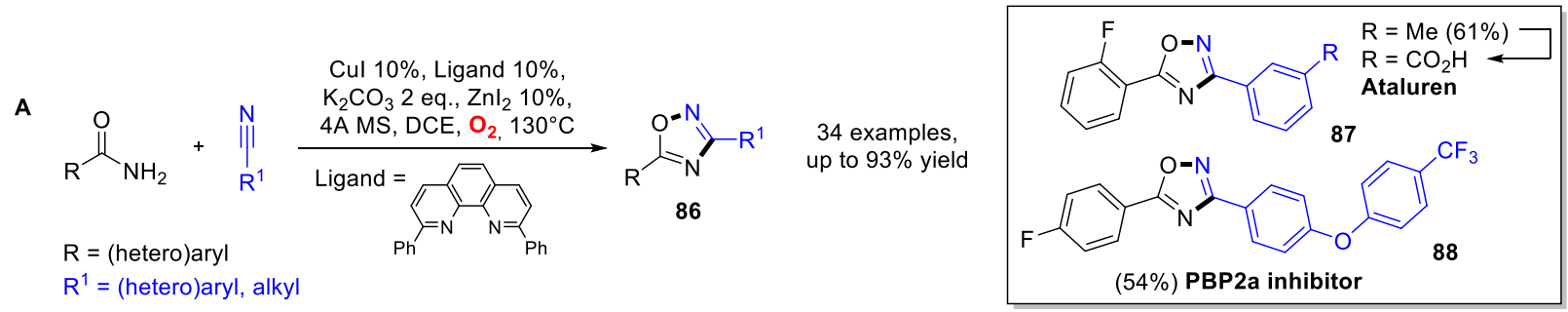

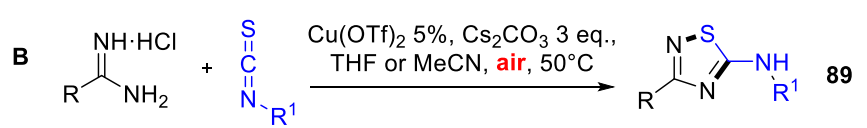

$\begin{array}{ll}R=\text { (hetero)aryl, Me, SBn } & 18 \text { examples intramol. (1 step), } \\ & 6 \text { examples intermol. (2 steps), }\end{array}$

$\mathrm{R}^{1}=$ aryl, alkyl

up to $86 \%$ yield

C ${ }_{\mathrm{NH}_{2}}^{\mathrm{N}}+\stackrel{\mathrm{I}}{\mathrm{I}}_{\mathrm{N}_{\mathrm{R}}}^{\mathrm{S}}$

$\mathrm{R}=$ aryl, alkyl, allyl

Cul $20 \%$, 2,4-dimethylpyridine 2 eq., $\mathrm{DCE}, \mathrm{O}_{2}, 50^{\circ} \mathrm{C}$

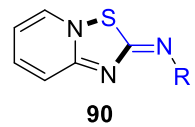

34 examples, up to $93 \%$ yield

D

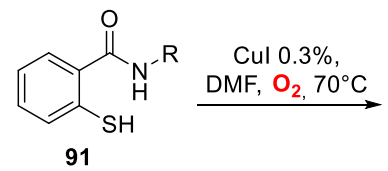

$\mathrm{R}=$ (hetero)aryl, alkyl, allyl

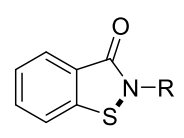

92

15 examples, up to $99 \%$ yield
I 20\%, dioxane,

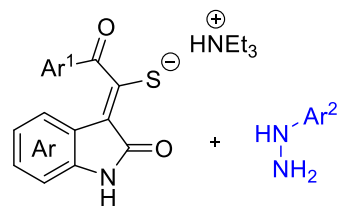

96 $\mathrm{O}_{2}, 100^{\circ} \mathrm{C}$

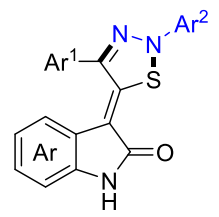

97

30 examples, up to $92 \%$ yield
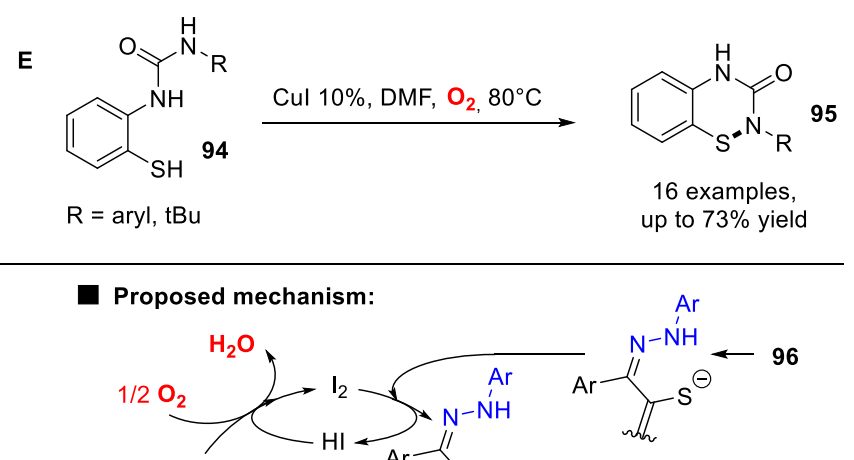

$$
\begin{aligned}
& \mathrm{Se} \text { (powder) } \\
& \mathrm{R}=\text { (hetero)aryl } \\
& \mathrm{R}^{1}=\text { aryl, alkyl }
\end{aligned}
$$

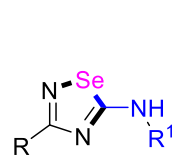

98

26 examples, up to $99 \%$ yield

Proposed mechanism:

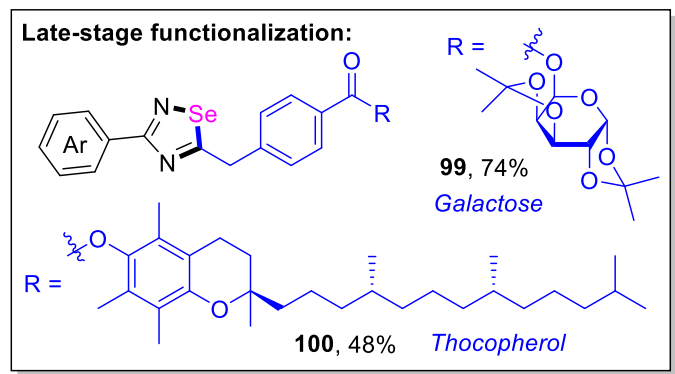

Scheme 14. Synthesis of various heterocyclic cores via oxidative N-O, N-S and N-Se bond formation. 
The addition of amidines to isothiocyanates, followed by intramolecular N-S coupling, results in 5-amino1,2,4-thiadiazoles 89, another important heterocyclic core (

Scheme 14B). ${ }^{66}$ Although the cascade process gave lower yields than the intramolecular coupling starting from the intermediate imidoylthioureas, the reaction could still be completed in short time (2-6 h) and without else than $\mathrm{Cu}(\mathrm{OTf})_{2}$ and $\mathrm{Cs}_{2} \mathrm{CO}_{3}$, required for the $\mathrm{N}-\mathrm{S}$ coupling. An extension of this protocol was recently reported by Jiang and $\mathrm{Wu}$, who employed 2-aminopyridines as substrates to synthesize thiadiazolopyridines 90 (

Scheme 14C). ${ }^{67}$ The reaction was fairly sensitive to the substitution pattern of both coupling partners, but high yields could be obtained for several examples.

Benzoisothiazolones $92^{68}$ and benzothiadiazinones $95^{69}$ can be prepared in high yields from 2mercaptobenzamides $\mathbf{9 1}$ or 2-mercaptophenylureas $\mathbf{9 4}$ under aerobic Cul catalysis, as demonstrated respectively by Kanai/Kuninobu and Dogan (

Scheme 14D-E). $\mathrm{N}$-aryl and $\mathrm{N}$-alkyl substituents are tolerated in both protocols, and one example of benzothiazetidine 93 was also reported in $84 \%$ yield. ${ }^{68}$

A couple of interesting metal-free protocols for similar couplings have also been reported. The synthesis of 1,2,3-thiadiazole derivatives 97 was reported by Li and Jiang using iodine as a SET catalyst (

Scheme 14F), ${ }^{70}$ while an oxidative N-Se bond formation strategy for the synthesis of 1,2,4-selenadiazoles 98 was recently disclosed by Ji/Wang and co-workers (

Scheme 14G). ${ }^{71}$ The reaction of isocyanides with selenium powder results in the corresponding isoselenocyanates 101, which undergo coupling with the amidines and oxidative cyclization, giving the selenadiazoles. Considering the lack of metal catalyst in this process, the role of oxygen was suggested to be related to the oxidation of the anionic organic intermediate $\mathbf{1 0 2}$ formed upon addition of the imidine to the isoselenocyanate, leading to a Se-centered radical (103). This radical might then undergo cyclization via different pathways (

Scheme 14). ${ }^{71}$

\section{Oxidative C-Heteroatom Bond Formation}

\subsection{Oxidative $\mathrm{C}-\mathrm{N}$ bond formation}

The formation of $\mathrm{Csp}^{2}-\mathrm{N}$ bonds through oxidative $\mathrm{C}-\mathrm{H}$ bond amination is a very common strategy for the synthesis of aza-heterocycles, and many examples have now been investigated under aerobic conditions. This transformation typically requires the use of transition metal catalysts, and many reports using $\mathrm{Pd}$ and other noble metals, as well as a variety of oxidants, were reported..$^{72}$ More recently, the use of $\mathrm{Cu}$ and other base metals have instead become predominant, and some procedures under metal-free conditions have also been successfully applied. Due to the many examples reported in the recent literature, only a selection will be reported in this section.

In 2008 Buchwald reported the synthesis of benzimidazoles 105 from amidines 104 using catalytic $\mathrm{Cu}(\mathrm{OAC})_{2}$ and acetic acid as additive under an $\mathrm{O}_{2}$ atmosphere (

Scheme 15A). ${ }^{73}$ Similar procedures were then employed for the synthesis of pyrido[1,2-a]benzimidazoles 107 by Zhu/Zhang ${ }^{74}$ and Maes, ${ }^{75}$ and for the synthesis of 1,4-dihydropyrazine moieties 109 by Fu ( 
Scheme 15B-C). ${ }^{76}$ The mechanism of this reaction is not certain, and different views are reported in the literature, but the role of the acid seems to be related to the formation of a more electrophilic $\mathrm{Cu}$ intermediate, as more electron-withdrawing carboxylates were shown to be more effective in some cases. ${ }^{75}$

Several other syntheses have been reported using similar protocols in the presence of a base. Examples are imidazobenzimidazoles 111 and benzoimidazoquinazolinones 113, prepared in high yields despite the long reaction time ( 2 days or more), as reported by Fu (

Scheme 15D-E). ${ }^{77-78}$

The Maes group reported the synthesis of purine derivatives 117 and 118 via aerobic formation of two different $\mathrm{C}-\mathrm{N}$ bonds. While the formation of the C4-N9 bond in

Scheme 15F-top required the use of a Fe catalyst in large amounts and $24 \mathrm{~h}$ reaction time in most cases, ${ }^{79}$ the $\mathrm{C} 5-\mathrm{N} 7$ bond in

Scheme 15F-bottom could be easily formed with $\mathrm{Cu}$, either using $\mathrm{O}_{2}$ ( $18 \mathrm{~h}$ reaction) or $\mathrm{tBu}_{2} \mathrm{O}_{2}$ ( $2 \mathrm{~h}$ reaction) as oxidant. ${ }^{80}$ Despite the longer reaction time required when using $\mathrm{O}_{2}$, the yields obtained in this case are often higher than those achieved with $\mathrm{tBu}_{2} \mathrm{O}_{2}$.

The synthesis of pyrazoles, indazoles, and similar compounds, which can be also prepared by oxidative N-N bond formation (Section 6.1), have been reported using this strategy, either under metal catalysis ${ }^{81-83}$ or in metal-free conditions ${ }^{84-85}$ (

Scheme 15G-H). Hydrazine derivatives need to be employed as starting materials in these cases, which have to be prepared beforehand. 
A

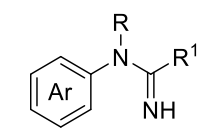

104

$\mathrm{R}=\mathrm{H}, \mathrm{Me}$

$\mathrm{R}^{1}=\mathrm{Ar}, \mathrm{tBu}$
$\mathrm{Cu}(\mathrm{OAc})_{2}$ 15\%, AcOH 5 eq.,

$\mathrm{DMSO}, \mathrm{O}_{2}, 100^{\circ} \mathrm{C}$

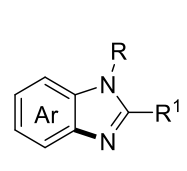

105

26 examples, up to $89 \%$ yield
B

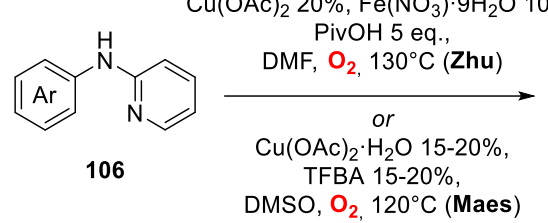

DMSO, $\mathrm{O}_{2}, 120^{\circ} \mathrm{C}$ (Maes)
19 examples, up to $85 \%$ yield

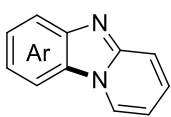

107

18 examples, up to $98 \%$ yield
$\mathrm{Ar}=\mathrm{EDG}$ and EWG-substituted (Maes)

$\mathrm{Ar}=\mathrm{EDG}$, limited EWG (Zhu)

TFBA $=3,4,5$-trifluorobenzoic acid

C

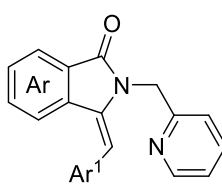

$\mathrm{Cu}\left(\mathrm{O}_{2} \mathrm{CCF}_{3}\right)_{2} 20 \%$

$\mathrm{PivOH} 3$ eq., DMF, air, $100-110^{\circ} \mathrm{C}$

108

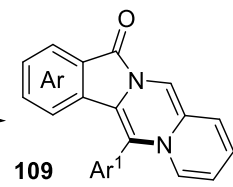

22 examples, up to $88 \%$ yield
(110)

$\mathrm{R}=\mathrm{Ar}$, alkyl

$\mathrm{R}^{1}=\mathrm{H}, \mathrm{Me}$
$\mathrm{Cu}(\mathrm{OAc})_{2} 20 \%$, phen $40 \%$, $\mathrm{NaOAc} 4$ eq., $m$-xylene, $\mathrm{O}_{2}, 155^{\circ} \mathrm{C}$<smiles>[R]c1ccc2c(c1)n1ccnc1n2[R]</smiles>

111

24 examples, up to $97 \%$ yield i) Cul or $\mathrm{CuBr} 20 \%$,
$\begin{gathered}\text { L-proline } 40 \%, \\ \text { Ullmann } \\ \text { coupling }\end{gathered}$

$\mathrm{Ar}=$ also pyridine $\mathrm{R}=$ alkyl
114
G

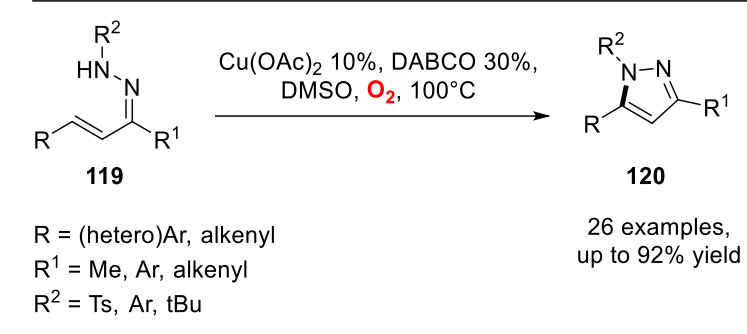

$\mathrm{FeCl}_{2} \cdot 4 \mathrm{H}_{2} \mathrm{O} 15-40 \%$, DMSO, $\mathrm{O}_{2}, 120^{\circ} \mathrm{C}$

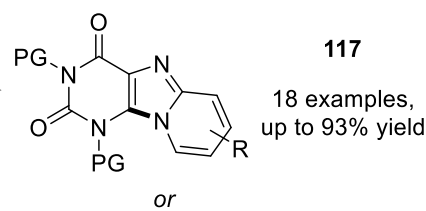

or

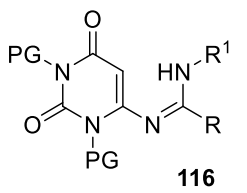

116

Fe cat:

$P G=p-m e t h o x y p h e n y l$

$R=E W G$ and $E D G$ in $3,4,5$ positions
DMSO, $\mathrm{N}_{2}, 120^{\circ} \mathrm{C}$

Cul $15 \%$, DMSO,

$\mathrm{O}_{2}, 120^{\circ} \mathrm{C}$
Cul $15 \%, \mathrm{tBu}_{2} \mathrm{O}_{2} 2$ eq.,

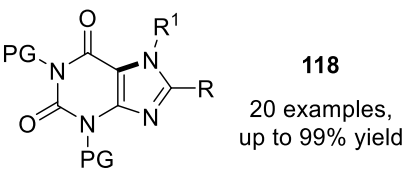

Cu cat:

$P G=M e, B n$

$\mathrm{R}=$ (hetero)Ar, alkyl

$\mathrm{R}^{1}=\mathrm{H}$, alkyl

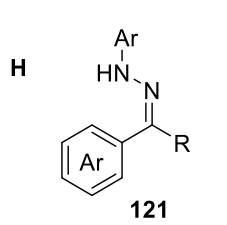

$\mathrm{Cu}(\mathrm{OAc})_{2} 10 \%$, DABCO $30 \%$,

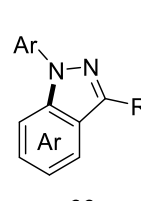

16 examples,

up to $86 \%$ yield

TEMPO $33 \%, \mathrm{NaHCO}_{3} 1$ eq.,

DMSO, $\mathrm{O}_{2}, 140^{\circ} \mathrm{C}$

66
45 examples, up to $95 \%$ yield

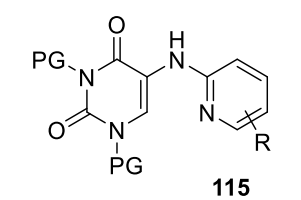

$\mathrm{R}=\mathrm{Ar}$, alkyl

Scheme 15. Synthesis of various aza-heterocycles via oxidative C-N bond formation.

Although no thorough mechanistic investigations have been yet reported for these transformations, several authors reported preliminary studies involving kinetic isotope effect (KIE), radical-trapping experiments, or electron paramagnetic spectroscopy (EPR) studies. On the basis of these, two main mechanisms have been proposed. One involves the formation of free $\mathrm{C}$ - or $\mathrm{N}$-centered radicals, and has been suggested for both metalfree and metal-catalyzed reactions. ${ }^{79,83-84}$ In the case of metal-catalyzed reactions no obvious coordination between metal and substrate has been suggested, and the metal seems to act mostly as an oxidant for a double single electron-transfer (SET) process, as described in Scheme 16-top. The second mechanism is often proposed only based on radical-trapping experiments. The absence of an inhibition is interpreted in terms of coordination of the two coupling moieties to the metal, (e.g. electrophilic aromatic substitution, or a different mechanism), and the absence of free radicals (Scheme 16-bottom). Variations of these mechanisms can be postulated for different substrates. 


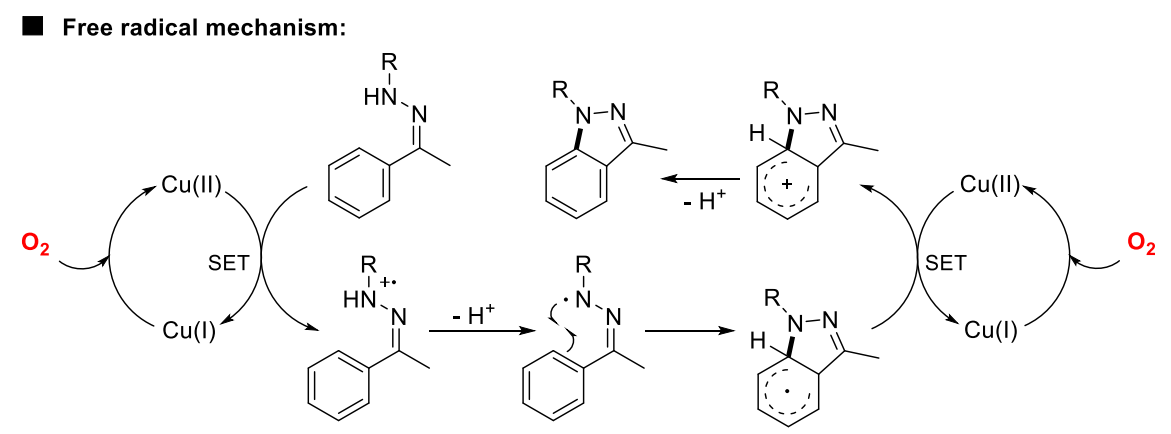

Non-radical mechanism:

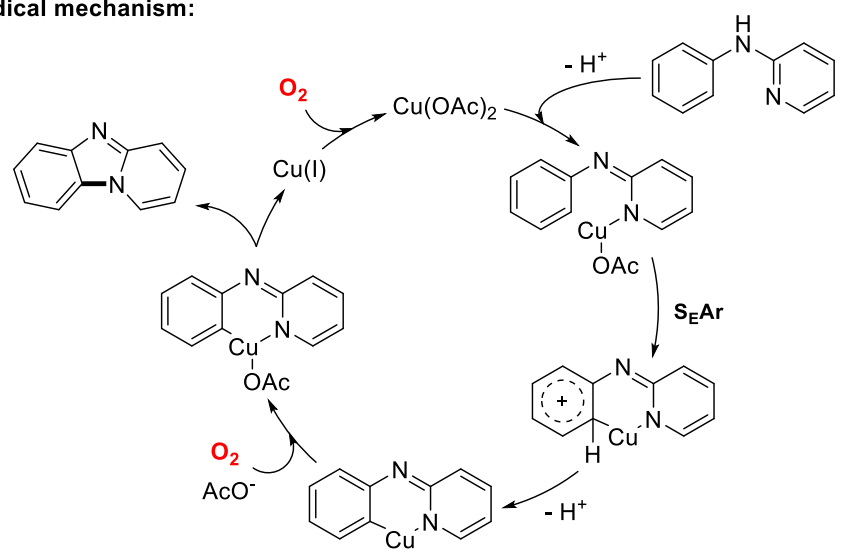

Scheme 16. Examples of radical and non-radical mechanisms proposed for the $\mathrm{Cu}$-catalyzed oxidative $\mathrm{C}-\mathrm{N}$ bond formation in heterocyclic synthesis.

\subsection{Oxidative $\mathrm{C}-\mathrm{O}$ and $\mathrm{C}-\mathrm{S}$ bond formation}

Oxidative cross-dehydrogenative couplings for the formation of $\mathrm{C}-\mathrm{O}$ and $\mathrm{C}-\mathrm{S}$ bonds in heterocycles have also been explored, albeit less frequently than $\mathrm{C}-\mathrm{N}$ bonds.

In particular, benzoxazoles have been prepared using a variety of protocols, which can be divided into two main strategies. In 2008 Nagasawa disclosed a Cu-catalyzed aerobic C-O coupling leading from N-phenylamides to benzoxazoles 122, according to Scheme $17 \mathrm{~A} .{ }^{86-87}$ Despite being fairly substrate-sensitive, the reaction proceeded well giving decent yields of a variety of 2-aryl and 2-alkyl benzoxazoles. The reaction was suggested to occur with a mechanism similar to the formation of benzimidazoles 105 reported by Buchwald in the same year (

Scheme 15A), i.e. via an electrophilic aromatic substitution as in Scheme 16-bottom. A second strategy involves, instead of the activation of an $\mathrm{Ar}-\mathrm{H}$ bond, the nucleophilic attack of an $\mathrm{OH}$ group to an imine. The required intermediate 123 can be formed in-situ from different substrates, ${ }^{88-90}$ as shown in Scheme 17B. Importantly, the use of $o$-aminothiols as substrates also proved amenable to cyclization, furnishing benzothiazoles 124 using Methods 1 and 2 (Scheme 17B). Note that Method 1 involves a strategy described in Section 2.1, but was reported here for comparison.

Isoxazoles 126 and 1,3,4-oxadiazoles 128 can also be synthesized via dehydrogenative C-O couplings, as shown by Sun ${ }^{91}$ and Patel, ${ }^{92}$ respectively (Scheme 17C-D).

A metal-free synthesis of 2-aminobenzothiazoles 130 starting from isothiocyanates 129 and a variety of amines (primary, secondary, aromatic) was developed by Fan and Zhang in 2017, employing iodine as catalyst (Scheme 17E). ${ }^{93}$ This protocol is particularly interesting, as it is complementary to the Pd-catalyzed insertion of 
isocyanides (cf. Section 3), which only allows the introduction of mono-substituted amino groups in the heterocycle (and mostly $\mathrm{N}$-alkyl substituents). The reaction was proposed to be initiated by the addition of the amine to the isothiocyanate, resulting in the intermediate thiourea 131, which then cyclizes upon formation of a S-I bond (Scheme 17E, right). This reaction worked similarly using other oxidants or stoichiometric iodine.

A

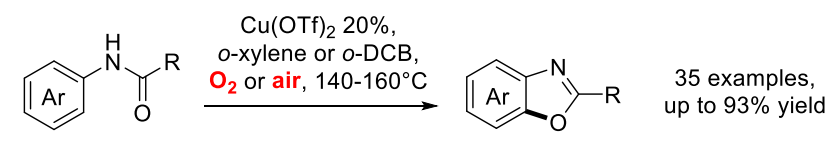

$\mathrm{R}=\mathrm{Ar}, \mathrm{alkyl}$

122

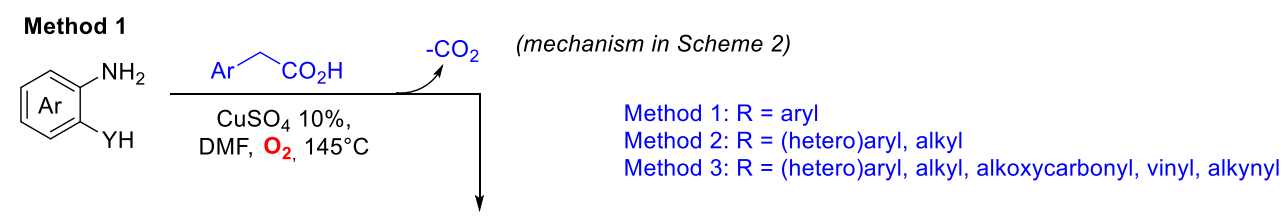

B<smiles>COc1ccccc1N</smiles>
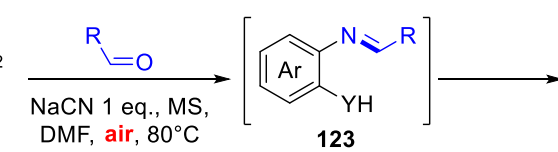

$\overbrace{122}^{\mathrm{Ar}} \mathrm{N}$ or

\section{Method 1:}

14 ex., up to $71 \%$ yield

Method 2:

12 ex., up to $86 \%$ yield

Method 3:

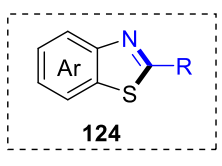

Method 1:

12 ex., up to $95 \%$ yield

Method 2:

1 ex., $71 \%$ yield
32 ex., up to $90 \%$ yield
C

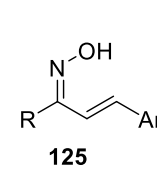

$\mathrm{R}=\mathrm{Ar}, \mathrm{Me}$
$\mathrm{Cu}(\mathrm{OAc})_{2} 10 \%$, DMSO, $\mathrm{O}_{2}, 100^{\circ} \mathrm{C}$

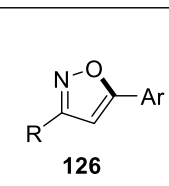

28 examples, up to $93 \%$ yield

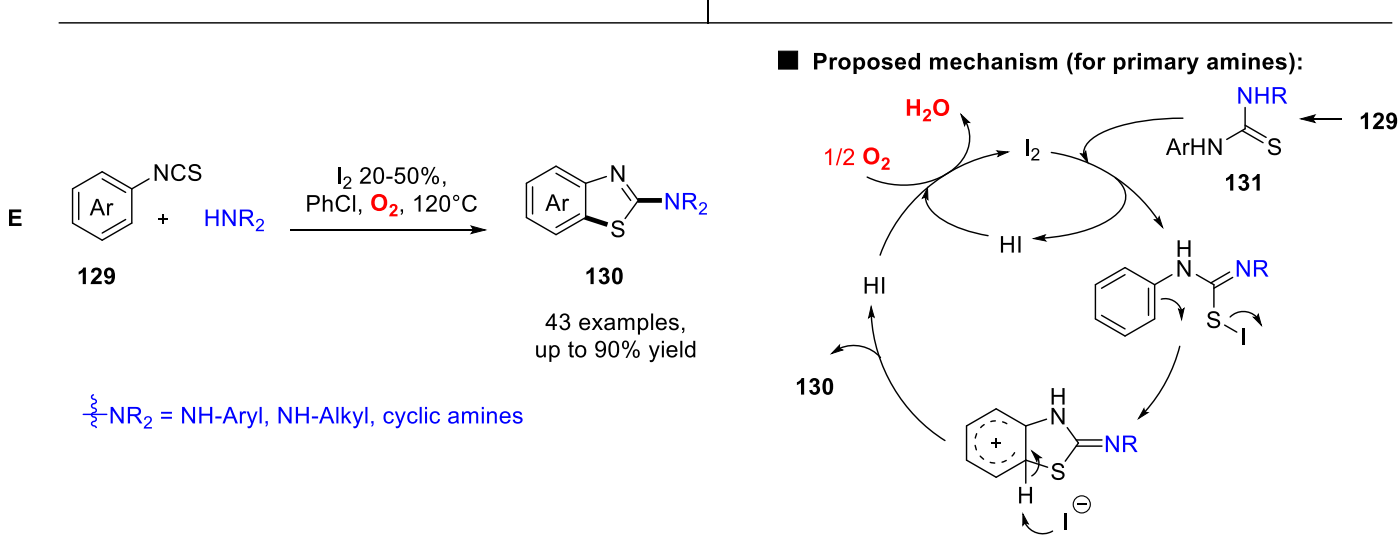

Scheme 17. Synthesis of various heterocycles via oxidative C-O and C-S bond formation.

\section{Intermolecular Oxidative Cyclization with Unsaturated Moieties}

A variety of substrates can be oxidatively coupled with unsaturated moieties leading to formal [3+2] cycloaddition products. As different mechanistic pathways can lead to the same overall transformation, these reactions are somewhat difficult to classify, and some of the processes presented in other sections might also fall in this category. Here a few specific strategies will be treated, divided according to substrate and mechanism. 


\subsection{Synthesis of imidazole derivatives via cyclization of amidines with electron-poor alkenes}

The aerobic coupling/cyclization of 2-aminopyridines with $\alpha, \beta$-unsaturated carbonyls to give 3-aroylimidazo[1,2a]pyridines 132 was first reported by Hajra in 2014 (

Scheme 18A). ${ }^{94}$ The reaction was catalyzed by $\mathrm{Cu}$ salts under an $\mathrm{O}_{2}$ atmosphere, and gave good yields (74-86\%) of the desired compounds with complete regioselectivity. The reaction was shown to proceed via a noncatalyzed Michael addition of the 2-aminopyridine to the $\alpha, \beta$-unsaturated carbonyl to give the intermediate shown in

Scheme 18-bottom, followed by an oxidative cyclization and aromatization. The main limitation of this protocol was the necessity to use chalcones (or dibenzylideneacetone) as coupling partners. Aliphatic unsaturated ketones did not give any product, and benzylideneacetone gave only $26 \%$ yield of the corresponding heterocycle. ${ }^{94}$ Other similar reports appeared in the following years, including a multicomponent process where the formation of the unsaturated carbonyl occurred in-situ via an aldol reaction, reported by Kumar, ${ }^{95}$ and the extension of the procedure to cynnamaldehydes by Bharate and co-workers (133,

Scheme 18B). ${ }^{96}$ Hachem and Gree subsequently further extended the scope to $\gamma$-difluoroenones 134 , albeit with limited yields (

Scheme 18C). ${ }^{97}$

In 2015 an alternative catalytic method for this reaction was reported by Li et al., who introduced the use of $\mathrm{Fe} / \mathrm{I}_{2}$ as a double catalyst for the cyclization of amidines with chalcones, furnishing imidazoles 138 (

Scheme 18D). ${ }^{98}$ The use of $\mathrm{I}_{2}$ as co-catalyst facilitates the formation of the second $\mathrm{C}-\mathrm{N}$ bond after the initial Michael addition. This made higher yields possible to achieve, and enlarged the substrate scope of the reaction. $\mathrm{Fe} / \mathrm{I}_{2}$ and $\mathrm{Cu} / \mathrm{I}_{2}$ catalytic systems were therefore subsequently employed for similar reactions. ${ }^{99-100}$ In particular, an interesting advancement was brought forward by Nguyen and co-workers, who performed the coupling of 2-aminopyridines and 2-aminopirimidines with cyclohexenones, previously not reactive in this type of transformation (139,

Scheme $18 \mathrm{E}) .{ }^{100}$ Notably, the use of $\mathrm{I}_{2}$ or Fe alone in this case only resulted in the formation of the Michael adduct, without cyclization taking place.

The cyclization of aminopyridines with nitroalkenes under photoredox catalysis by Eosin Y, reported in 2016 by Singh and co-workers, is a variation of the reaction with chalcones described above. Under these conditions, however, a reversed selectivity was observed, and 3-arylimidazopyridines $\mathbf{1 4 0}$ were obtained instead of the corresponding 2-aryl derivatives (

Scheme 18F). ${ }^{101}$ This reaction was also suggested to occur via a Michael addition (pyridine as nucleophile), although the origin of the different regioselectivity is not clear. 


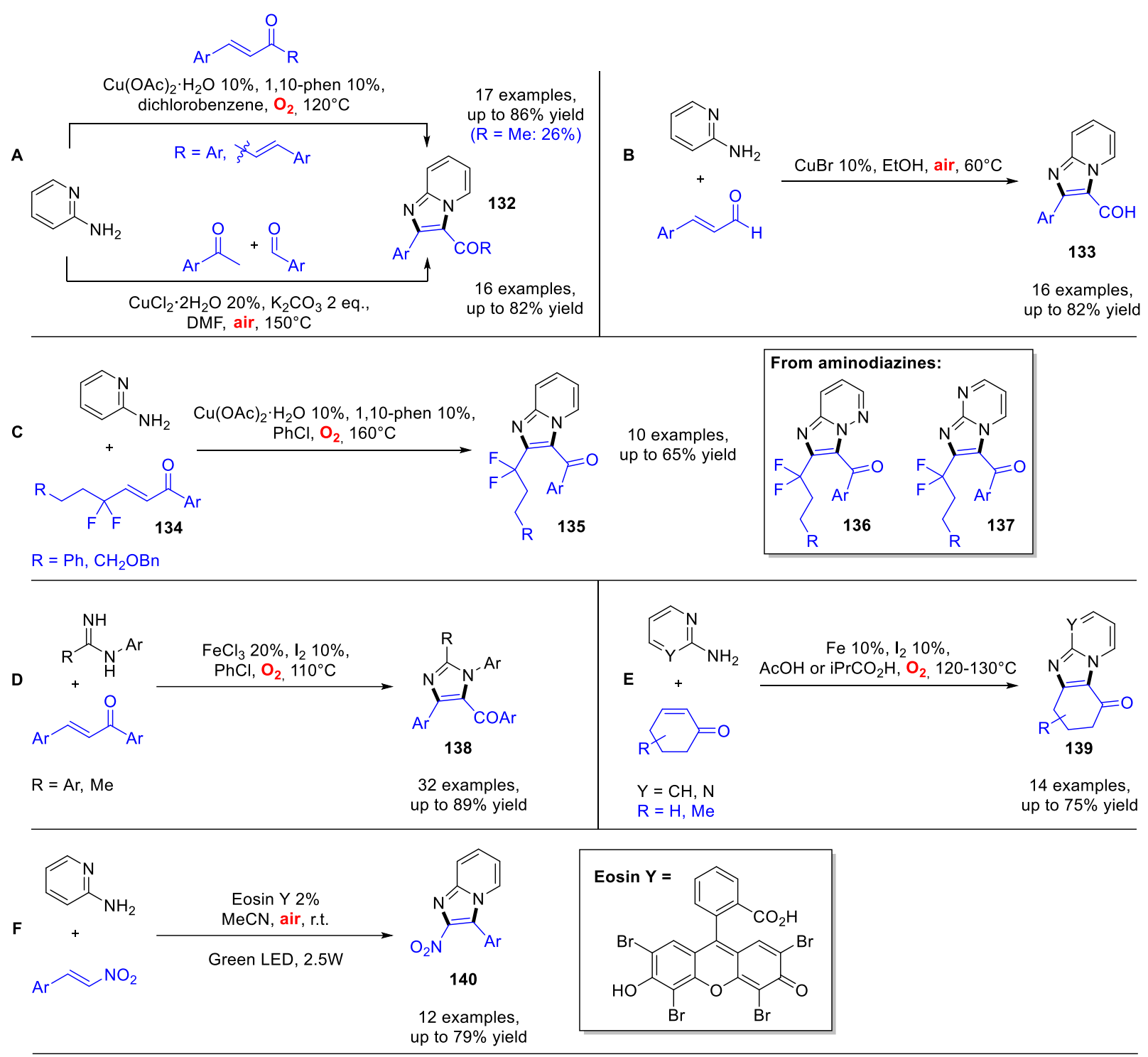

General mechanism for the cyclization via Michael addition:

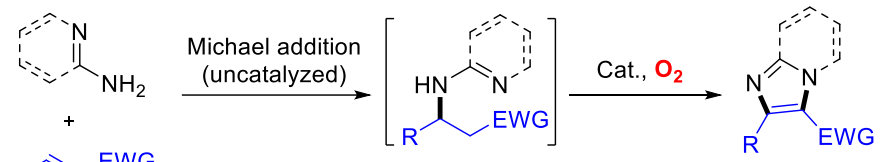

R $\curvearrowright W G$

Scheme 18. Cyclization of amidines/aminopyridines with electron-poor alkenes for the synthesis of imidazole and pyridoimidazole derivatives.

\subsection{Synthesis of benzofurans via cyclization of phenols with alkynes}

The synthesis of benzofurans via oxidative cyclization of phenols and alkynes has been known for quite some time. This reaction can be performed in different ways, for example in the presence of a transition metal catalyst with stoichiometric $\mathrm{Cu}$ as oxidant, ${ }^{102-103}$ or using $N$-phenoxyamides as internal oxidant, ${ }^{104}$ but some interesting examples have been disclosed that make use of $\mathrm{O}_{2}$ in the presence of catalytic $\mathrm{Cu}$ or $\mathrm{Au}$.

A catalytic amount of $\mathrm{Cu}$ (II) salt and a stoichiometric amount of $\mathrm{ZnCl}_{2}$ as Lewis acid under an $\mathrm{O}_{2}$ atmosphere was demonstrated by Jiang to be a useful combination for this reaction in $2013 .{ }^{105}$ The reaction performed 
efficiently with symmetric diarylalkynes and with arylalkylalkynes, in the latter case in a regioselective way (141 and 142,

Scheme 19A). The same transformation was later reported by Guo using only a cationic Au(I) catalyst, affording similar results. ${ }^{106}$ Important follow-up research on this transformation revealed that $\mathrm{ZnCl}_{2}$ alone can promote the oxidative cyclization acting as both Lewis acid and oxidant in the absence of other reagents. ${ }^{107}$

Satoh and Miura recently reported the synthesis of benzofurans from salicylaldehydes via an Ir-catalyzed decarbonylative coupling with alkynes (

Scheme 19B). ${ }^{108}$ This reaction occurs with a completely different mechanism from the above coupling, furnishing overall much lower yields and requiring more complex reaction mixtures. Nonetheless, a dialkylalkyne could be coupled with a $29 \%$ yield, and the reaction with unsymmetric arylalkylalkyne gave the opposite regioisomer to the one obtained by Jiang with $\mathrm{Cu} / \mathrm{ZnCl}_{2}$ (cf. 142 and 143).

A

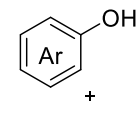

$\mathrm{Cu}(\mathrm{OTf})_{2} 10 \%, \mathrm{ZnCl}_{2} 1.5$ eq.,

$\mathrm{R}=\mathrm{R}$

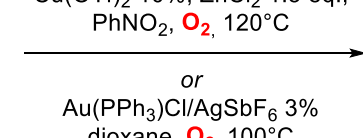

$\mathrm{Cu} / \mathrm{ZnCl}{ }_{2}$

25 examples,

up to $93 \%$ yield
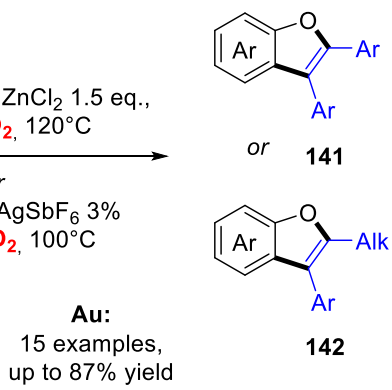

or 141

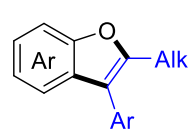

142
Proposed mechanism:

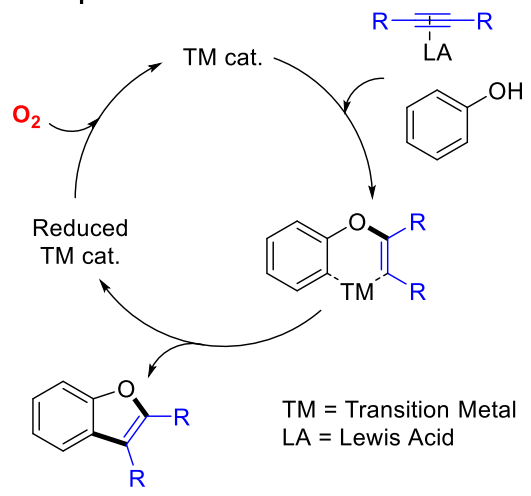

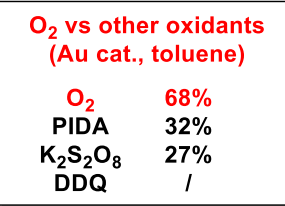

DDQ I
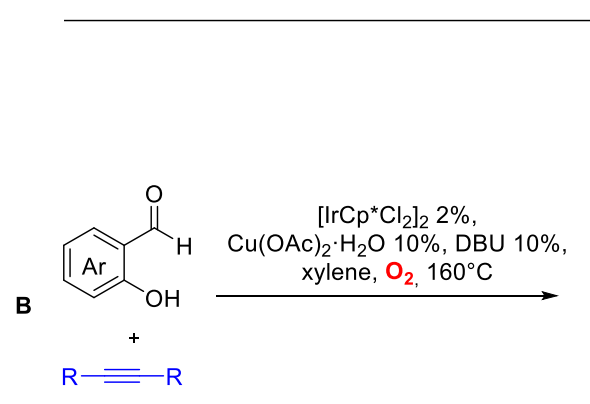
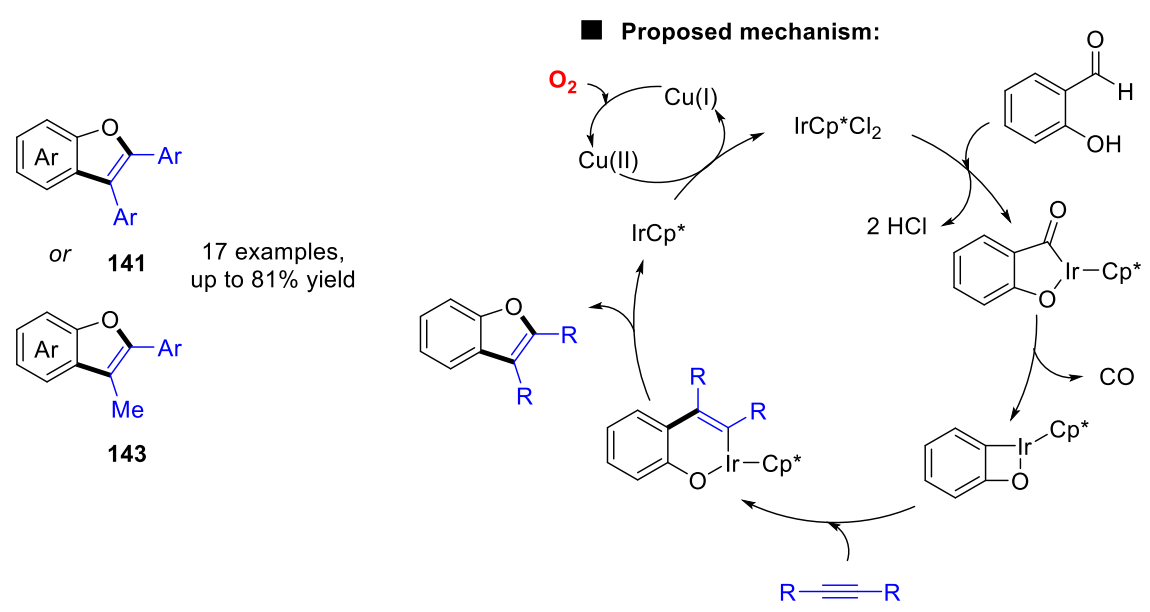

Scheme 19. Formation of benzofurans via aerobic cyclization of alkynes with phenols or salicylaldehydes.

\subsection{Miscellaneous cyclizations with alkynes}

Halogenated imidazopyridines 144 and related compounds $145-148$ could be prepared in good yields $(60-80 \%)$ by cyclization of 2-aminopyridines with haloalkynes (

Scheme 20A). ${ }^{109}$ As haloalkynes are prone to react via nucleophilic substitution/addition, or to dimerization, this is a remarkable transformation. Either iodo, bromo, or chloroalkynes proved suitable for this transformation, and a variety of aminopyridines and aminodiazines could be efficiently coupled. A non-radical mechanism was 
suggested for this reaction, involving migratory insertion of the alkyne into a $\mathrm{N}$-Cu bond, followed by reductive elimination. ${ }^{109}$

A

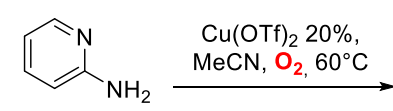

$\mathrm{R}=\mathrm{x}$

$\mathrm{R}=$ aryl, alkyl

$\mathrm{X}=\mathrm{I}, \mathrm{Br}, \mathrm{Cl}$

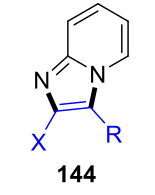

25 examples, up to $80 \%$ yield

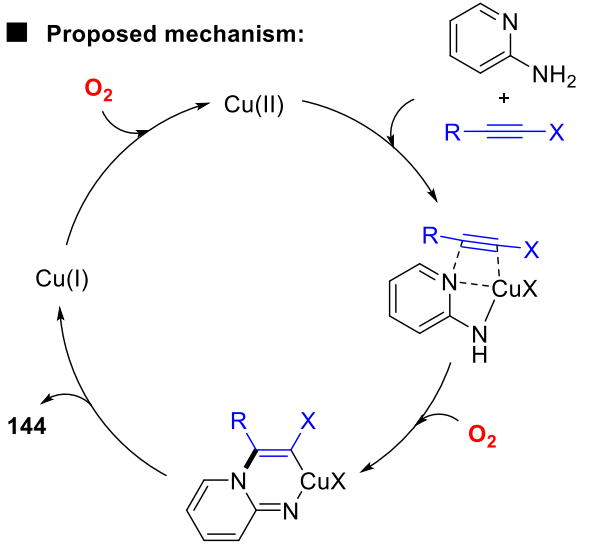

Proposed mechanism:

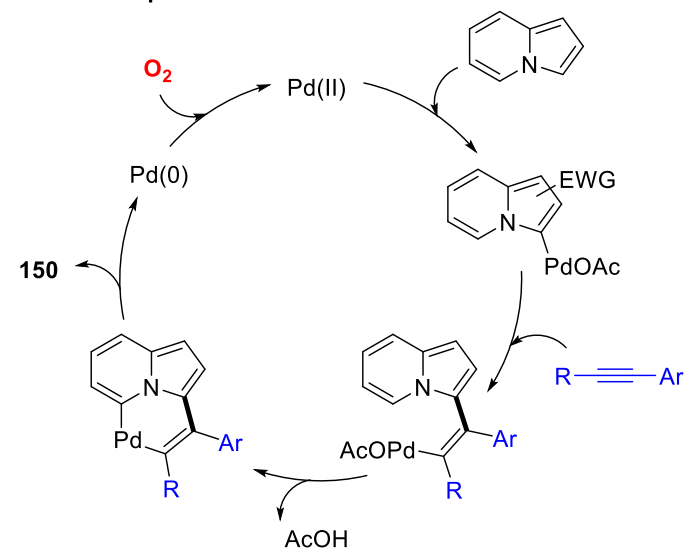

Proposed mechanism:

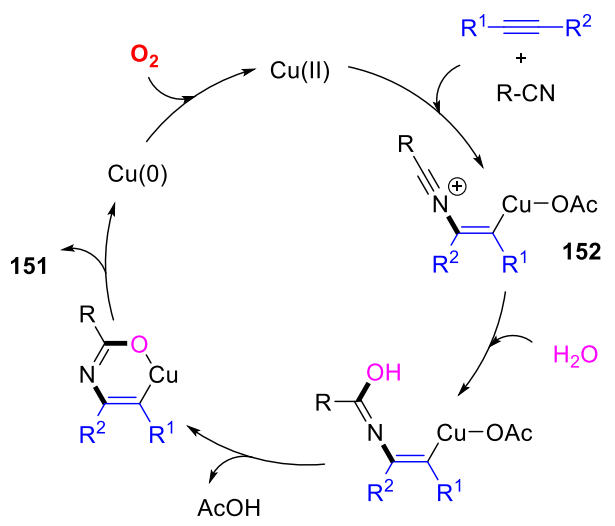

Scheme 20. Formation of various 5-membered heterocycles via aerobic cyclization of alkynes with different substrates.

$\mathrm{Hu}$ and co-workers reported an interesting synthesis of pyrrolo[2,1,5-cd]indolizines $\mathbf{1 5 0}$ via cyclization between indolizines 149 and diarylalkynes under Pd catalysis (

Scheme 20B). ${ }^{110}$ The substrate scope for this reaction is limited to electron-poor indolizines (EWG in position 2 or 3 ) and to diarylalkynes (unsymmetrical alkynes give regioisomeric mixtures). 
The synthesis of trisubstituted oxazoles 151 via a formal [2+2+1] cycloaddition between alkynes, nitriles and water under Cu catalysis was disclosed by Jiang in 2012 (

Scheme 20C). ${ }^{111}$ Both diaryl and dialkyl alkynes were well tolerated, and regioselective products were obtained with unsymmetrical alkynes; moreover, a variety of nitriles (allylic, aromatic and aliphatic) could be incorporated into the final products. Although the mechanism was not studied in detail, some experiments suggested an initial nucleophilic attack of the nitrile to the Cu-activated alkyne, followed by nucleophilic attack of water to the cationic intermediate 152, and finally intramolecular C-O bond formation. ${ }^{111}$ As for other reactions of this kind (Section 7), the role of oxygen is the re-oxidation of the reduced Cu species at the end of the catalytic cycle.

\section{Conclusions}

This review summarizes some of the recent developments in the synthesis of heterocycles making use of catalytic aerobic oxidation. Such oxidative processes can be applied to the formation of C-C, C-heteroatom, and heteroatom-heteroatom bonds, sometimes contributing to the formation of more than one bond at once. Because of this, a very large array of moieties can be accessed with these methods, and N-, O-, S-, and Secontaining heterocycles can be easily prepared, often with good yields and selectivity. The generally broad scope, combined with the low price and toxicity, and the high atom-economic nature of oxygen make it a very versatile and appealing reagent for the synthesis of heterocycles, when compared to other commonly used oxidants (e.g. hypervalent iodine species, peroxides, stoichiometric Cu or Ag salts).

The current state of the research in aerobic oxidation led to the identification of specific catalytic systems, such as $\mathrm{Cu} / \mathrm{O}_{2}, \mathrm{Fe} / \mathrm{O}_{2}$ or $\mathrm{I}_{2} / \mathrm{O}_{2}$, which can be applied, with relatively little variation, to a number of processes, as can be seen in this review. These systems are now also being studied from a mechanistic perspective, and can be considered a starting point for future aerobic oxidation research projects.

As mentioned in the introduction, practical limitations to the use of gaseous $\mathrm{O}_{2}$ exist, which somewhat prevent its use in the fine chemicals industry. Nonetheless, research in this field is very active, and adequate technology for the broader and safer use of aerobic oxidation processes might become available in the near future.

\section{Acknowledgements}

The author acknowledges the European Union for a Marie Curie European post-doctoral fellowship (FlowAct, Grant No. 794072).

\section{References}

1. Allen, S. E.; Walvoord, R. R.; Padilla-Salinas, R.; Kozlowski, M. C. Chem. Rev. 2013, 113, 6234-6458. https://doi.org/10.1021/cr300527g

2. Sterckx, H.; Morel, B.; Maes, B. U. Angew. Chem. Int. Ed., doi:10.1002/anie.201804946. https://doi.org/10.1002/anie.201804946 
3. Stahl, S. S.; Alster, P. L. Liquid Phase Aerobic Oxidation Catalysis: Industrial Applications and Academic Perspectives. Wiley-VCH: 2016, 10.1002/9783527690121.

https://doi.org/10.1002/9783527690121

4. Hone, C. A.; Roberge, D. M.; Kappe, C. O. ChemSusChem 2017, 10, 32-41. https://doi.org/10.1002/cssc.201601321

5. Gemoets, H. P. L.; Su, Y.; Shang, M.; Hessel, V.; Luque, R.; Noël, T. Chem. Soc. Rev. 2016, 45, 83-117. https://doi.org/10.1039/C5CS00447K

6. Pieber, B.; Kappe, C. O. In Organometallic Flow Chemistry, Noël, T., Ed. Springer International Publishing: Cham, 2016, 10.1007/3418_2015_133 pp 97-136.

7. Yaragorla, S.; Vijaya Babu, P. Tetrahedron Lett. 2017, 58, 1879-1882. https://doi.org/10.1016/i.tetlet.2017.04.007

8. Yang, L.; Shi, X.; Hu, B. Q.; Wang, L. X. Asian J. Org. Chem. 2016, 5, 494-498. https://doi.org/10.1002/ajoc.201600041

9. Li, Q.; Huang, Y.; Chen, T.; Zhou, Y.; Xu, Q.; Yin, S.-F.; Han, L.-B. Org. Lett. 2014, 16, 3672-3675. https://doi.org/10.1021/ol501454j

10. Chen, X.; Chen, T.; Ji, F.; Zhou, Y.; Yin, S.-F. Catal. Sci. Technol. 2015, 5, 2197-2202. https://doi.org/10.1039/C4CY01618A

11. Sterckx, H.; De Houwer, J.; Mensch, C.; Caretti, I.; Tehrani, K. A.; Herrebout, W. A.; Van Doorslaer, S.; Maes, B. U. W. Chem. Sci. 2016, 7, 346-357. https://doi.org/10.1039/C5SC03530A

12. Pang, X.; Wu, M.; Ni, J.; Zhang, F.; Lan, J.; Chen, B.; Yan, R. J. Org. Chem. 2017, 82, 10110-10120. https://doi.org/10.1021/acs.joc.7b01575

13. Pereira, M. d. F.; Thiéry, V. Org. Lett. 2012, 14, 4754-4757. https://doi.org/10.1021/ol302006b

14. Li, J.; Zhang, J.; Yang, H.; Gao, Z.; Jiang, G. J. Org. Chem. 2017, 82, 765-769. https://doi.org/10.1021/acs.joc.6b02501

15. Tan, Z.; Zhao, H.; Zhou, C.; Jiang, H.; Zhang, M. J. Org. Chem. 2016, 81, 9939-9946. https://doi.org/10.1021/acs.joc.6b02117

16. Cai, Z.-J.; Wang, S.-Y.; Ji, S.-J., Org. Lett. 2012, 14, 6068-6071. https://doi.org/10.1021/ol302955u

17. Wang, Y.-F.; Chen, H.; Zhu, X.; Chiba, S. J. Am. Chem. Soc. 2012, 134, 11980-11983. https://doi.org/10.1021/ja305833a

18. Pan, J.; Li, X.; Qiu, X.; Luo, X.; Jiao, N. Org. Lett. 2018, 20, 2762-2765. https://doi.org/10.1021/acs.orglett.8b00992

19. Xu, Z.; Zhang, C.; Jiao, N. Angew. Chem. Int. Ed. 2012, 51, 11367-11370. https://doi.org/10.1002/anie.201206382

20. Vlaar, T.; Ruijter, E.; Maes, B. U. W.; Orru, R. V. A. Angew. Chem. Int. Ed. 2013, 52, 7084-7097. https://doi.org/10.1002/anie.201300942

21. Lang, S., Chem. Soc. Rev. 2013, 42, 4867-4880. https://doi.org/10.1039/c3cs60022j

22. Wang, Y.; Wang, H.; Peng, J.; Zhu, Q. Org. Lett. 2011, 13, 4604-4607. https://doi.org/10.1021/ol201807n

23. Vidyacharan, S.; Murugan, A.; Sharada, D. S. J. Org. Chem. 2016, 81, 2837-2848. https://doi.org/10.1021/acs.joc.6b00048 
24. Vlaar, T.; Cioc, R. C.; Mampuys, P.; Maes, B. U. W.; Orru, R. V. A.; Ruijter, E. Angew. Chem. Int. Ed. 2012, 51, 1305813061.

https://doi.org/10.1002/anie.201207410

25. Liu, B.; Yin, M.; Gao, H.; Wu, W.; Jiang, H. J. Org. Chem. 2013, 78, 3009-3020.

https://doi.org/10.1021/jo400002f

26. Vlaar, T.; Bensch, L.; Kraakman, J.; Velde, C. M. L. V.; Maes, B. U. W.; Orru, R. V. A.; Ruijter, E. Adv. Synth. Catal. 2014, 356, 1205-1209.

https://doi.org/10.1002/adsc.201301129

27. Vlaar, T.; Orru, R. V. A.; Maes, B. U. W.; Ruijter, E. J. Org. Chem. 2013, 78, 10469-10475. https://doi.org/10.1021/jo401924h

28. Vidyacharan, S.; Chaitra, N. C.; Sagar, A.; Sharada, D. S. Synth. Comm. 2015, 45, 898-907. https://doi.org/10.1080/00397911.2014.992076

29. Ji, F.; Lv, M.-F.; Yi, W.-B.; Cai, C. Org. Biomol. Chem. 2014, 12, 5766-5772. https://doi.org/10.1039/C4OB00484A

30. Sambiagio, C.; Schönbauer, D.; Blieck, R.; Dao-Huy, T.; Pototschnig, G.; Schaaf, P.; Wiesinger, T.; Zia, M. F.; WencelDelord, J.; Besset, T.; Maes, B. U. W.; Schnürch, M. Chem. Soc. Rev. 2018, 47, 6603-6743. https://doi.org/10.1039/C8CS00201K

31. Liu, Y.-J.; Xu, H.; Kong, W.-J.; Shang, M.; Dai, H.-X.; Yu, J.-Q. Nature 2014, 515, 389. https://doi.org/10.1038/nature13885

32. Tereniak, S. J.; Stahl, S. S. J. Am. Chem. Soc. 2017, 139, 14533-14541. https://doi.org/10.1021/jacs.7b07359

33. Dang, Y.; Deng, X.; Guo, J.; Song, C.; Hu, W.; Wang, Z.-X. J. Am. Chem. Soc. 2016, 138, 2712-2723. https://doi.org/10.1021/jacs.5b12112

34. Gadge, S. T.; Bhanage, B. M. RSC Advances 2014, 4, 10367-10389. https://doi.org/10.1039/c3ra46273k

35. Gabriele, B.; Mancuso, R.; Salerno, G. Eur. J. Org. Chem. 2012, 2012, 6825-6839. https://doi.org/10.1002/ejoc.201200794

36. Noujima, A.; Mitsudome, T.; Mizugaki, T.; Jitsukawa, K.; Kaneda, K. Green Chem. 2013, 15, 608-611. https://doi.org/10.1039/c2gc36851j

37. Mancuso, R.; Raut, D. S.; Marino, N.; De Luca, G.; Giordano, C.; Catalano, S.; Barone, I.; Andò, S.; Gabriele, B. Chem. Eur. J. 2016, 22, 3053-3064. https://doi.org/10.1002/chem.201504443

38. Acerbi, A.; Carfagna, C.; Costa, M.; Mancuso, R.; Gabriele, B.; Della Ca', N. Chem. Eur. J. 2018, 24, 4835-4840. https://doi.org/10.1002/chem.201706067

39. Mancuso, R.; Raut, D. S.; Della Ca', N.; Fini, F.; Carfagna, C.; Gabriele, B. ChemSusChem 2015, 8, 2204-2211. https://doi.org/10.1002/cssc.201500343

40. Chen, Y.; Hone, C. A.; Gutmann, B.; Kappe, C. O. Org. Process Res. Dev. 2017, 21, 1080-1087. https://doi.org/10.1021/acs.oprd.7b00217

41. Cheng, C.-C.; Yan, S.-J., In Organic Reactions, Dauben, W. G. Ed. John Wiley \& Sons: 1982; Vol. 28, pp 37-201. https://doi.org/10.1002/0471264180.or028.02

42. Shiri, M.; Zolfigol, M. A.; Kruger, H. G.; Tanbakouchian, Z. In Advances in Heterocyclic Chemistry, Katritzky, A. R., Ed. Academic Press: 2011; Vol. 102, pp 139-227.

43. Senadi, G. C.; Dhandabani, G. K.; Hu, W.-P.; Wang, J.-J. Green Chem. 2016, 18, 6241-6245. https://doi.org/10.1039/C6GC02396G 
44. Wan-Lu, M.; Meirong, W.; Hui-Jing, L.; Deng-Ming, H.; Yi-Yun, Z.; Chao-Yi, L.; Ying, L.; Yan-Chao, W. Adv. Synth. Catal. 2017, 359, 4250-4257. https://doi.org/10.1002/adsc.201700715

45. Xiangui, C.; Yanjun, X.; Cheng, L.; Fuhong, X.; Guo-Jun, D. Eur. J. Org. Chem. 2017, 2017, 577-581.

46. Neumann, J. J.; Suri, M.; Glorius, F. Angew. Chem. Int. Ed. 2010, 49, 7790-7794, and refs. therein. https://doi.org/10.1002/anie.201002389

47. Mohan, D. C.; Ravi, C.; Rao, S. N.; Adimurthy, S. Org. Biomol. Chem. 2015, 13, 3556-3560. https://doi.org/10.1039/C50B00018A

48. Fustero, S.; Simón-Fuentes, A.; Sanz-Cervera, J. F. Org. Prep. Proced. Int. 2009, 41, 253-290. https://doi.org/10.1080/00304940903077832

49. Suri, M.; Jousseaume, T.; Neumann, J. J.; Glorius, F. Green Chem. 2012, 14, 2193-2196. https://doi.org/10.1039/c2gc35476d

50. Tang, X.; Huang, L.; Yang, J.; Xu, Y.; Wu, W.; Jiang, H. Chem. Comm. 2014, 50, 14793-14796. https://doi.org/10.1039/C4CC06747A

51. Wu, Q.; Zhang, Y.; Cui, S. Org. Lett. 2014, 16, 1350-1353. https://doi.org/10.1021/ol500094w

52. Yu, D.-G.; Suri, M.; Glorius, F. J. Am. Chem. Soc. 2013, 135, 8802-8805. https://doi.org/10.1021/ja4033555

53. Peng, J.; Xie, Z.; Chen, M.; Wang, J.; Zhu, Q. Org. Lett. 2014, 16, 4702-4705. https://doi.org/10.1021/ol502010g

54. Chen, C.-y.; Tang, G.; He, F.; Wang, Z.; Jing, H.; Faessler, R. Org. Lett. 2016, 18, 1690-1693. https://doi.org/10.1021/acs.orglett.6b00611

55. Cheng-yi, C.; Fengxian, H.; Guangrong, T.; Han, D.; Zhaobin, W.; Dawei, L.; Lujiang, D.; Roger, F. Eur. J. Org. Chem. 2017, 2017, 6604-6608.

56. Ueda, S.; Nagasawa, H. J. Am. Chem. Soc. 2009, 131, 15080-15081. https://doi.org/10.1021/ja905056z

57. Bartels, B.; Bolas, C. G.; Cueni, P.; Fantasia, S.; Gaeng, N.; Trita, A. S. J. Org. Chem. 2015, 80, 1249-1257. https://doi.org/10.1021/j0502536t

58. Wang, F.; You, Q.; Wu, C.; Min, D.; Shi, T.; Kong, Y.; Zhang, W. RSC Advances 2015, 5, 78422-78426. https://doi.org/10.1039/C5RA15919A

59. Xu, H.; Jiang, Y.; Fu, H. Synlett 2013, 24, 125-129.

60. Sudheendran, K.; Schmidt, D.; Frey, W.; Conrad, J.; Beifuss, U. Tetrahedron 2014, 70, 1635-1645. https://doi.org/10.1016/j.tet.2014.01.019

61. Xu, H.; Ma, S.; Xu, Y.; Bian, L.; Ding, T.; Fang, X.; Zhang, W.; Ren, Y. J. Org. Chem. 2015, 80, 1789-1794. https://doi.org/10.1021/j0502709t

62. Tasuku, H.; Satoshi, U.; Takahiro, O.; Norihiko, T.; Kensuke, O.; Hideko, N. Chem. Eur. J. 2014, 20, 4156-4162. https://doi.org/10.1002/chem.201302997

63. Guru, M. M.; Punniyamurthy, T. J. Org. Chem. 2012, 77, 5063-5073. https://doi.org/10.1021/jo300592t

64. Wu, L.; Guo, S.; Wang, X.; Guo, Z.; Yao, G.; Lin, Q.; Wu, M. Tetrahedron Lett. 2015, 56, 2145-2148. https://doi.org/10.1016/j.tetlet.2015.03.019

65. Rao, K. M.; Gyum, K. W.; Kyungjae, M.; You, H. S. Eur. J. Org. Chem. 2016, 2016, 438-442.

66. Kim, H.-Y.; Kwak, S. H.; Lee, G.-H.; Gong, Y.-D. Tetrahedron 2014, 70, 8737-8743. https://doi.org/10.1016/i.tet.2014.09.023 
67. Yu, W.; Huang, Y.; Li, J.; Tang, X.; Wu, W.; Jiang, H. J. Org. Chem. 2018, 83, 9334-9343. https://doi.org/10.1021/acs.joc.8b01292

68. Wang, Z.; Kuninobu, Y.; Kanai, M. J. Org. Chem. 2013, 78, 7337-7342. https://doi.org/10.1021/jo401056g

69. Doğan, Ş. D. Tetrahedron 2017, 73, 2217-2224. https://doi.org/10.1016/i.tet.2017.02.063

70. Fan, W.; Li, Q.; Li, Y.; Sun, H.; Jiang, B.; Li, G. Org. Lett. 2016, 18, 1258-1261. https://doi.org/10.1021/acs.orglett.6b00079

71. Fang, Y.; Zhu, Z.-L.; Xu, P.; Wang, S.-Y.; Ji, S.-J. Green Chem. 2017, 19, 1613-1618. https://doi.org/10.1039/C6GC03521C

72. Henry, M. C.; Mostafa, M. A. B.; Sutherland, A. Synthesis 2017, 49, 4586-4598. https://doi.org/10.1055/s-0036-1588536

73. Brasche, G.; Buchwald, S. L. Angew. Chem. Int. Ed. 2008, 47, 1932-1934. https://doi.org/10.1002/anie.200705420

74. Wang, H.; Wang, Y.; Peng, C.; Zhang, J.; Zhu, Q. J. Am. Chem. Soc. 2010, 132, 13217-13219. https://doi.org/10.1021/ja1067993

75. Masters, K.-S.; Rauws, T. R. M.; Yadav, A. K.; Herrebout, W. A.; Van der Veken, B.; Maes, B. U. W. Chem. Eur. J. 2011, 17, 6315-6320. https://doi.org/10.1002/chem.201100574

76. Lu, J.; Jin, Y.; Liu, H.; Jiang, Y.; Fu, H. Org. Lett. 2011, 13, 3694-3697. https://doi.org/10.1021/ol2013395

77. Wang, X.; Jin, Y.; Zhao, Y.; Zhu, L.; Fu, H. Org. Lett. 2012, 14, 452-455. https://doi.org/10.1021/ol202884z

78. Xu , H.; Fu , H., Chem. Eur. J. 2012, 18, 1180-1186. https://doi.org/10.1002/chem.201102794

79. Maes, J.; Rauws, T. R. M.; Maes, B. U. W., Chem. Eur. J. 2013, 19, 9137-9141. https://doi.org/10.1002/chem.201301248

80. Morel, B.; Franck, P.; Bidange, J.; Sergeyev, S.; Smith, D. A.; Moseley, J. D.; Maes, B. U. W. ChemSusChem 2017, 10, 624-628.

https://doi.org/10.1002/cssc.201601483

81. Hu, J.; Chen, S.; Sun, Y.; Yang, J.; Rao, Y., Org. Lett. 2012, 14, 5030-5033. https://doi.org/10.1021/ol3022353

82. Li, X.; He, L.; Chen, H.; Wu, W.; Jiang, H., J. Org. Chem. 2013, 78, 3636-3646. https://doi.org/10.1021/jo400162d

83. Lan, C.; Tian, Z.; Liang, X.; Gao, M.; Liu, W.; An, Y.; Fu, W.; Jiao, G.; Xiao, J.; Xu, B. Adv. Synth. Catal. 2017, 359, 3735-3740.

https://doi.org/10.1002/adsc.201700669

84. Hu, J.; Xu, H.; Nie, P.; Xie, X.; Nie, Z.; Rao, Y., Chem. Eur. J. 2014, 20, 3932-3938. https://doi.org/10.1002/chem.201304923

85. Yu, J.; Lim, J. W.; Kim, S. Y.; Kim, J.; Kim, J. N., Tetrahedron Lett. 2015, 56, 1432-1436. https://doi.org/10.1016/j.tetlet.2015.01.183

86. Ueda, S.; Nagasawa, H., Angew. Chem. Int. Ed. 2008, 47, 6411-6413. https://doi.org/10.1002/anie.200801240

87. Ueda, S.; Nagasawa, H., J. Org. Chem. 2009, 74, 4272-4277. 
https://doi.org/10.1021/jo900513z

88. Cho, Y. H.; Lee, C.-Y.; Ha, D.-C.; Cheon, C.-H. Adv. Synth. Catal. 2012, 354, 2992-2996.

https://doi.org/10.1002/adsc.201200684

89. Yang, D.; Yan, K.; Wei, W.; Tian, L.; Shuai, Y.; Li, R.; You, J.; Wang, H. Asian J. Org. Chem. 2014, 3, 969-973. https://doi.org/10.1002/ajoc.201402085

90. Chen, X.; Ji, F.; Zhao, Y.; Liu, Y.; Zhou, Y.; Chen, T.; Yin, S.-F. Adv. Synth. Catal. 2015, 357, 2924-2930. https://doi.org/10.1002/adsc.201500515

91. Sun, Y.; Abdukader, A.; Zhang, H.; Yang, W.; Liu, C., RSC Advances 2017, 7, 55786-55789. https://doi.org/10.1039/C7RA11436B

92. Guin, S.; Ghosh, T.; Rout, S. K.; Banerjee, A.; Patel, B. K. Org. Lett. 2011, 13, 5976-5979. https://doi.org/10.1021/ol202409r

93. Xu, Y.; Li, B.; Zhang, X.; Fan, X., J. Org. Chem. 2017, 82, 9637-9646. https://doi.org/10.1021/acs.joc.7b01683

94. Kamarul, M.; Avik, K. B.; Subhajit, M.; Adinath, M.; Alakananda, H., Adv. Synth. Catal. 2014, 356, 1105-1112. https://doi.org/10.1002/adsc.201300900

95. Kaswan, P.; Pericherla, K.; Saini, H. K.; Kumar, A., RSC Advances 2015, 5, 3670-3677. https://doi.org/10.1039/C4RA13056A

96. Bharate, J. B.; Abbat, S.; Bharatam, P. V.; Vishwakarma, R. A.; Bharate, S. B., Org. Biomol. Chem. 2015, 13, 77907794.

https://doi.org/10.1039/C5OB00776C

97. Hariss, L.; Hadir, K. B.; El-Masri, M.; Roisnel, T.; Grée, R.; Hachem, A., Beilstein J. Org. Chem. 2017, 13, $2115-2121$. https://doi.org/10.3762/bjoc.13.208

98. Zhu, Y.; Li, C.; Zhang, J.; She, M.; Sun, W.; Wan, K.; Wang, Y.; Yin, B.; Liu, P.; Li, J. Org. Lett. 2015, 17, 3872-3875. https://doi.org/10.1021/acs.orglett.5b01854

99. Li, J. J.; Song, C.; Cui, D.-M.; Zhang, C., Org. Biomol. Chem. 2017, 15, 5564-5570. https://doi.org/10.1039/C7OB01018D

100. Nguyen, T. B.; Corbin, M.; Retailleau, P.; Ermolenko, L.; Al-Mourabit, A., Org. Lett. 2015, 17, 4956-4959. https://doi.org/10.1021/acs.orglett.5b02340

101. Yadav, S.; Srivastava, M.; Rai, P.; Tripathi, B. P.; Mishra, A.; Singh, J.; Singh, J., New J. Chem. 2016, 40, 9694-9701. https://doi.org/10.1039/C6NJ02365G

102. Kuram, M. R.; Bhanuchandra, M.; Sahoo, A. K. Angew. Chem. Int. Ed. 2013, 52, 4607-4612. https://doi.org/10.1002/anie.201210217

103. Zhu, R.; Wei, J.; Shi, Z., Chem. Sci. 2013, 4, 3706-3711. https://doi.org/10.1039/c3sc51489g

104. Liu, G.; Shen, Y.; Zhou, Z.; Lu, X., Angew. Chem. Int. Ed. 2013, 52, 6033-6037. https://doi.org/10.1002/anie.201300881

105. Zeng, W.; Wu, W.; Jiang, H.; Huang, L.; Sun, Y.; Chen, Z.; Li, X., Chem. Comm. 2013, 49, 6611-6613. https://doi.org/10.1039/c3cc42326c

106. Liao, J.; Guo, P.; Chen, Q., Catal. Commun. 2016, 77, $22-25$. https://doi.org/10.1016/i.catcom.2016.01.009

107. Sreenivasulu, C.; Gopi Krishna Reddy, A.; Satyanarayana, G., Org. Chem. Front. 2017, 4, 972-977. https://doi.org/10.1039/C6Q000531D

108. Yamane, S.; Hinoue, T.; Usuki, Y.; Itazaki, M.; Nakazawa, H.; Hayashi, Y.; Kawauchi, S.; Miura, M.; Satoh, T. Chem. Eur. J. 2018, 24, 7852-7855. 
https://doi.org/10.1002/chem.201801245

109. Gao, Y.; Yin, M.; Wu, W.; Huang, H.; Jiang, H., Adv. Synth. Catal. 2013, 355, 2263-2273. https://doi.org/10.1002/adsc.201300157

110. Hu, H.; Li, G.; Hu, W.; Liu, Y.; Wang, X.; Kan, Y.; Ji, M., Org. Lett. 2015, 17, 1114-1117. https://doi.org/10.1021/ol503681n

111. Li, X.; Huang, L.; Chen, H.; Wu, W.; Huang, H.; Jiang, H., Chem. Sci. 2012, 3, 3463-3467. https://doi.org/10.1039/c2sc21041j

\section{Author's Biography}

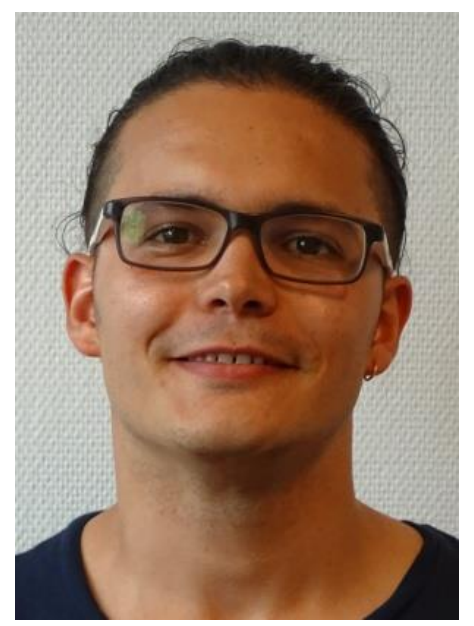

Carlo Sambiagio studied chemistry at the University of Cagliari (IT), acquiring his Master's degree in 2011. He then moved to the University of Leeds (UK), completing his PhD on Cu-catalyzed cross-couplings in 2015, under the supervision of Prof. Patrick McGowan. After that he spent two years as a postdoc in Prof. Bert Maes's group in Antwerp (BE), mostly exploring aerobic oxidation chemistry. Carlo has recently moved to the Eindhoven University of Technology (NL) as a EU Marie Curie fellow to work on flow chemistry with Prof. Timothy Noël. His interests generally include metal-catalyzed transformations and the development of new methodologies for organic synthesis. 